\title{
Smoothness and Non-Smoothness of the Fundamental Solution of Time Dependent Schrödinger Equations
}

\section{Kenji Yajima}

Department of Mathematical Sciences, University of Tokyo, Komaba, Meguroku, Tokyo 153, Japan

Received: 2 August 1995/Accepted: 16 April 1996

Abstract: The fundamental solution $E(t, s, x, y)$ of time dependent Schrödinger equations $i \partial u / \partial t=-(1 / 2) \triangle u+V(t, x) u$ is studied. It is shown that

- $E(t, s, x, y)$ is smooth and bounded for $t \neq s$ if the potential is sub-quadratic in the sense that $V(t, x)=o\left(|x|^{2}\right)$ at infinity;

- in one dimension, if $V(t, x)=V(x)$ is time independent and super-quadratic in the sense that $V(x) \geqq C(1+|x|)^{2+\varepsilon}$ at infinity, $C>0$ and $\varepsilon>0$, then $E(t, s, x, y)$ is nowhere $C^{1}$.

The result is explained in terms of the limiting behavior as the energy tends to infinity of the corresponding classical particle.

\section{Introduction}

We consider the time dependent Schrödinger equation with a real potential $V(t, x)$ :

$$
i \partial u / \partial t=-(1 / 2) \triangle u+V(t, x) u, \quad(t, x) \in \mathbf{R}^{1} \times \mathbf{R}^{m} .
$$

The equation generates a unique unitary propagator $\{U(t, s):-\infty<t, s<\infty\}$ in $L^{2}\left(\mathbf{R}^{m}\right)$ under the conditions to be imposed below and $u(t, x)=(U(t, s) \phi)(x)$ represents a unique solution of (1.1) which satisfies the initial condition $u(s, x)=\phi(x) \in$ $L^{2}\left(\mathbf{R}^{m}\right)$. Standard arguments show $U(t, s)$ is a two parameter family of strongly continuous unitary operators satisfying the semi-group properties: $U(t, t)=1$ and $U(t, s) U(s, r)=U(t, r)$. We denote by $E(t, s, x, y)$ the distribution kernel of $U(t, s)$ : $E=E(t, s, x, y)$ is the fundamental solution of Eq. (1.1), or FDS for short. In this paper, we show that

1. $E(t, s, x, y)$ is smooth and bounded with respect to $(x, y)$ for any $t \neq s$, provided $V$ is "sub-quadratic" in the sense that for all $|\alpha|=2, \lim _{|x| \rightarrow \infty}\left|\partial_{x}^{\alpha} V(t, x)\right|=0$ uniformly with respect to $t \in \mathbf{R}^{1}$;

2. in one dimension, if $V(t, x)=V(x)$ is time independent and "super-quadratic" in the sense that $V(x) \geqq C(1+|x|)^{2+\varepsilon}$ at infinity, $C>0$ and $\varepsilon>0$, then $E(t, s, x, y)$ is nowhere $C^{1}$. 
To state our main theorems more precisely, we introduce some notation. Let $\partial_{j}=\partial / \partial x_{j}, D_{j}=-i \partial_{j}, j=1, \ldots, m, \partial=\left(\partial_{1}, \ldots, \partial_{m}\right), D=\left(D_{1}, \ldots, D_{m}\right)$, and for the multi-index $\alpha=\left(\alpha_{1}, \ldots, \alpha_{m}\right), D^{\alpha}=D_{1}^{\alpha_{1}} \cdots D_{m}^{\alpha_{m}}, \partial^{\alpha}=\partial_{1}^{\alpha_{1}} \cdots \partial_{m}^{\alpha_{m}} \cdot|\alpha|=\alpha_{1}+\cdots+\alpha_{m}$. For a function $F, \partial F(x)$ denotes the gradient vector and $\partial^{2} F(x)$ denotes the Hessian matrix. For $\sigma \in \mathbf{R}, \mathscr{L}_{1}^{\sigma}\left(\mathbf{R}^{m}\right)$ is the Bessel space

$$
\mathscr{L}_{1}^{\sigma}\left(\mathbf{R}^{m}\right)=\left\{f \in \mathscr{S}^{\prime}\left(\mathbf{R}^{m}\right):(1-\triangle)^{\sigma / 2} f \in L^{1}\left(\mathbf{R}^{m}\right)\right\}
$$

and $\mathscr{L}_{1, \text { loc }}^{\sigma}\left(\mathbf{R}^{m}\right)$ is its localization. We say $f$ is nowhere in $\mathscr{L}_{1, \text { loc }}^{\sigma}\left(\mathbf{R}^{m}\right)$ if $\phi f \notin \mathscr{L}_{1}^{\sigma}\left(\mathbf{R}^{m}\right)$ for any $\phi \in C_{0}^{\infty}\left(\mathbf{R}^{m}\right)$. Note that $C_{0}^{1}\left(\mathbf{R}^{m}\right) \subset \mathscr{L}_{1}^{\sigma}\left(\mathbf{R}^{m}\right)$ for all $\sigma<1$. Finally the pair of functions $(x(t, s, y, k), p(t, s, y, k))$ always denotes the solution of Hamilton's equations corresponding to $(1.1)$ :

$$
\left\{\begin{array} { l } 
{ d x / d t = p ( t ) , } \\
{ d p / d t = - ( \partial _ { x } V ) ( t , x ( t ) ) , }
\end{array} \quad \left\{\begin{array}{l}
x(s, s, y, k)=y, \\
p(s, s, y, k)=k
\end{array}\right.\right.
$$

Theorem 1.1. Assume that $V(t, x)$ is $C^{\infty}$ with respect to $x \in \mathbf{R}^{m}$ and $\partial_{x}^{\alpha} V(t, x)$ is $C^{\sigma}$ with respect to $(t, x)$ for all $\alpha$, where $\sigma=0,1, \ldots$. Suppose that

$$
\lim _{|x| \rightarrow \infty} \sup _{t \in \mathbf{R}}\left|\partial_{x}^{\alpha} V(t, x)\right|=0, \quad \text { if }|\alpha|=2, \quad \text { and } \quad\left|\partial_{x}^{\alpha} V(t, x)\right| \leqq C_{\alpha}, \quad \text { for all }|\alpha| \geqq 3 .
$$

Then $E(t, s, x, y)$ is $C^{\infty}$ with respect to $(x, y)$ and all derivatives $\partial_{x}^{\alpha} \partial_{y}^{\beta} E(t, s, x, y)$ are $C^{\sigma+1}$ with respect to $(t, s, x, y)$ for $t \neq s$. Moreover, for every $T>0$, there exists a constant $C_{T}>0$ such that the following statements are satisfied. Write $\Omega_{T}=\left\{(t, s, x, y) \in \mathbf{R}^{2} \times \mathbf{R}^{2 m}: 0<|t-s| \leqq T,|x|^{2}+|y|^{2} \geqq C_{T}^{2}\right\}$.

1. For $(t, s, x, y) \in \Omega_{T}$, there exists a unique $k \in \mathbf{R}^{m}$ such that $x=x(t, s, y, k)$. The function $S(t, s, x, y)$ defined on $\Omega_{T}$ by

$$
S(t, s, x, y)=\int_{s}^{t}\left\{(1 / 2) p(\tau, s, y, k)^{2}-V(\tau, x(\tau, s, y, k))\right\} d \tau
$$

is smooth with respect to $(x, y)$ and all derivatives $\partial_{x}^{\alpha} \partial_{y}^{\beta} S(t, s, x, y)$ are $C^{\sigma+1}$ with respect to $(t, s, x, y)$. If $|\alpha+\beta| \geqq 2$, the following estimates are satisfied:

$$
\left|\partial_{x}^{\alpha} \partial_{y}^{\beta}\left\{S(t, s, x, y)-(x-y)^{2} /(2(t-s))\right\}\right| \leqq C_{\alpha \beta T}|t-s| .
$$

2. In $\Omega_{T}, E(t, s, x, y)$ may be written in the form

$$
E(t, s, x, y)=(2 \pi i(t-s))^{-m / 2} a(t, s, x, y) e^{i S(t, s, x, y)},
$$

where $a(t, s, x, y)$ satisfies, for all $\alpha$ and $\beta$, the estimates

$$
\left|\partial_{x}^{\alpha} \partial_{y}^{\beta} a(t, s, x, y)\right| \leqq C_{\alpha \beta T} .
$$

3. There exists a constant $T(V)$ such that, for $T \leqq T(V), C_{T}$ may be set equal to zero.

In the next theorem, we assume that $V(t, x)=V(x)$ is independent of $t$. Then $E(t, s, x, y)$ depends only on $(t-s, x, y)$ and we write $E(t, x, y)=E(t, 0, x, y)$. 
Theorem 1.2. Let $m=1$ and $V \in C^{3}\left(\mathbf{R}^{1}\right)$ be real valued. Assume further that outside a compact interval $K$ the following two conditions are met:

1. $V^{\prime \prime}(x)>0$ and $x V^{\prime}(x) \geqq 2 c V(x)>0$ for some $c>1$;

2. For $j=1,2,3, V^{(j)}(x)=O(1 / x) V^{(j-1)}(x)$ as $|x| \rightarrow \infty$.

Then, as a function of $(t, x, y), E(t, x, y)$ is nowhere in $\mathscr{L}_{1, \text { loc }}^{1 / 2 c}\left(\mathbf{R}^{3}\right)$. In particular, it is nowhere $C^{1}$.

Several remarks are in order.

Remark 1. We should supplement Theorem 1.2 by the following two statements:

1. For almost all $y \in \mathbf{R}^{1}$ (resp. $\left.x \in \mathbf{R}^{1}\right), E(t, x, y)$ is nowhere in $\mathscr{L}_{1, \text { loc }}^{1 / 2 c}\left(\mathbf{R}^{2}\right)$ with respect to $(t, x)$ (resp. $(t, y))$.

2. If $V(x)$ is $C^{\infty}$ and satisfies $V(x) \leqq C|x|^{2 c}$ for large $|x|$, in addition to the conditions of Theorem 1.2, then for any $\Phi \in C_{0}^{\infty}\left(\mathbf{R}^{3}\right)$, the Fourier transform $\widehat{\Phi E}$ of $\Phi E$ decays at infinity as follows: $|\widehat{\Phi E}(\tau, \xi, \eta)| \leqq C\left(1+|\tau|+|\xi|^{2}+|\eta|^{2}\right)^{-1 / 2 c}$. The proof of Theorem 1.2 shows that $-1 / 2 c$ is the best possible decay rate.

We shall give a proof of this remark.

Remark 2. When $T$ is small, i.e. $T \leqq T(V)$, Theorem 1.1 is well-known (see Fujiwara [6], and also Yajima [18] for an extension to the case where magnetic fields are present). Moreover, the results in [6] (and [18]) are proven for small $T>0$ under an assumption weaker than (1.3), viz, $\left|\partial_{x}^{\alpha} V(t, x)\right| \leqq C_{\alpha}$ for $|\alpha| \geqq 2$. For these potentials, however, $E(t, s, x, y)$ is in general not smooth for larger values of $|t-s|$. When $V(x)=x^{2} / 2$, Mehler's formula [13] shows that

$$
E(t, x, y)=\frac{1}{(2 \pi i \sin t)^{m / 2}} e^{(i / 2 \sin t)\left\{\left(|x|^{2}+|y|^{2}\right) \cos t-2 x \cdot y\right\}},
$$

from which one sees explicitly that $E$ is smooth when $t \neq n \pi, n \in \mathbf{Z}$, but is singular when $t=n \pi$. This "recurrence of singularities" takes places for a wide range of perturbations of $x^{2} / 2$ (cf. Zelditch [21] and Kapitanski-Rodnianski-Yajima [9]).

Remark 3. Zelditch's paper mentioned above also shows that if $V(t, x)$ is bounded with all $x$-derivatives, then FDS is smooth with respect to $(x, y)$ when $t \neq s,-\infty<t$, $s<\infty$, and can be written in the form

$$
E(t, s, x, y)=(2 \pi i(t-s))^{-m / 2} e^{i(x-y)^{2} / 2(t-s)} a(t, s, x, y)
$$

with $a(t, s, \cdot, \cdot) \in C^{\infty}$ as above. The proof that $E$ is smooth has been extended by Craig, Kappeler and Strauss [4] to the sub-linear potentials, $\left|\partial_{x}^{\alpha} V(x)\right| \leqq$ $C_{\alpha}(1+|x|)^{1-|\alpha|-\varepsilon}$ for all $\alpha, \varepsilon>0$, but they do not construct the structure formula like (1.6) or (1.9). The smoothness of the FDS can also be studied by investigating the smoothing property of the propagator $U(t, s)$. In this direction, we mention the works of Ozawa [14] and Yamazaki [20] and the references therein. After submission of this paper, we learned that Kapitanski-Rodnianski [8] have demonstrated the smoothness of $E(t, s, x, y)$ for a slightly different class of sub-quadratic potentials. 
Remark 4. The FDS of (1.1) can be very singular. If $H=-d^{2} / d x^{2}$ is the Dirichlet Laplacian on the interval $[0, \pi]$, which may be thought of as an extreme superquadratic case, then, the FDS $E$ is given by $E(t, x, y)=(2 / \pi) \sum_{n=1}^{\infty} e^{-i t n^{2}} \sin n x \sin n y$, which is nowhere locally integrable. Indeed, this is a direct consequence of the proof of Theorem 1.2 given below. Thus it is somewhat surprising that, for this $H$, the propagator of (1.1) still has a rather strong smoothing property,

$$
\left(\int_{0}^{2 \pi}\|u(t, \cdot)\|_{L^{4}([0, \pi])}^{4} d t\right)^{1 / 4} \leqq C\|\varphi\|_{L^{2}([0, \pi])}
$$

(cf. Zygmund [22]). Note however that the solution is much smoother in the whole space (cf. e.g. Yajima [19]) in the sense

$$
\left(\int_{0}^{\infty}\|u(t, \cdot)\|_{L^{\infty}(\mathbf{R})}^{4} d t\right)^{1 / 4} \leqq C\|\varphi\|_{L^{2}(\mathbf{R})} .
$$

For every $(s, y), E(t, s, x, y)$ is a solution of (1.1) with initial condition $E(s, s, x, y)$ $=\delta(x-y)$, Dirac's measure at the point $x=y$, and Theorems 1 and 2 may be considered, partly, as statements on the propagation of singularities for $E(t, s, x, y)$. Thus one may be tempted to think that the statements are consequences of Hörmander's celebrated theorem on propagation of singularities (cf. $[7,16]$ ), viz, the wave front set $W F(u)$ of the solution of the partial differential equation $Q(x, D) u=$ $\sum_{|\alpha| \leqq n} a_{\alpha}(x) D^{\alpha} u=0$ with real principal symbol $Q_{n}(x, \xi)=\sum_{|\alpha|=n} a_{\alpha}(x) \xi^{\alpha}$ is contained in the characteristic set $\left\{(x, \xi): Q_{n}(x, \xi)=0\right\}$ and is invariant under the Hamiltonian flow generated by the principal symbol. Note, however, that the principal symbol of the Schrödinger Eq. (1.1) is $\xi^{2} / 2$ and the characteristic set is $\left\{(t, x, \tau, \xi): t, \tau \in \mathbf{R}, x \in \mathbf{R}^{m}, \xi=0 \in \mathbf{R}^{m}\right\},(\tau, \xi)$ being the conjugate variables of $(t, x)$. Hence, each point $(t, x, \tau, 0)$ is a stationary point of the Hamiltonian flow of the principal symbol $\xi^{2} / 2$ and Hörmander's theorem unfortunately provides little information about the propagation of singularities for solutions of (1.1). (In this connection, see Craig, Kappelar and Strauss [4] and Kapitanski and Safarov [10].)

This situation has been analyzed further by Lascar [12] and Sakurai [15]. They introduced the notion of the quasi-homogeneous wave front set $W F^{a}(u)$ and showed how the set propagates for solutions of quasi-homogeneous (pseudo-)differential equations. Their theory, when applied to (1.1), shows that $W F^{a}(u)$ is contained in $\left\{(t, x, \tau, \xi): \tau=\xi^{2} / 2\right\}$ and is invariant under the Hamilton flow generated by the principal symbol $\xi^{2} / 2$ on each plane $t=$ constant. This implies that $E(t, s, x, y)$, as a function of $(t, x)$, is singular everywhere on the plane $t=s$ for every fixed $(s, y)$, however, it still does not tell us whether or not the singularity propagates in the forward or backward direction of time $t$.

Our results may be best "understood" if we believe in the following "conjecture": The singularities of the solution (defined in terms of a suitably modified notion of the wave front set) of the evolution equation $i \partial u / \partial t=P(t, x, D) u$ with real symbol $P(t, x, \xi)$ propagate along the limit set, as the energy tends to infinity, of the trajectories of $(t, x(t), \tau(t), p(t))$ of the Hamilton equations, not for the principal, but for the full symbol:

$$
d x / d t=\partial P / \partial p, \quad d p / d t=-\partial P / \partial x, \quad d \tau / d t=-\partial P / \partial t .
$$

In other words, the singularities propagate along trajectories with infinite energy. 
Let us "explain" our theorems from this point of view in one dimension when $V(t, x)=V(x)$ does not depend on $t$ and $V(x) \rightarrow \infty$ as $|x| \rightarrow \infty$, so that all trajectories of (1.2) are periodic functions of $t$.

When $V(x)=x^{2} / 2$, the period of the trajectories is independent of energy and is always equal to $2 \pi$. If $t \neq n \pi$, therefore, for any two points $x, y \in \mathbf{R}^{m}$ no solutions of (1.2) with $x(0)=y$ and very large $|p(0)|$ approach $x$ at time $t$. Hence the FDS is everywhere smooth when $t \neq n \pi$. On the other hand, if $t$ is an integer times half the period, i.e. $t=n \pi$, all trajectories leaving $y$ at time 0 reach $(-1)^{n} y$ at time $n \pi$ with momentum $(-1)^{n}$ times the initial one. Hence, for any $(n \pi, x, y)$, there is a sequence of trajectories $\left(t, x_{k}(t), \tau_{k}(t), p_{k}(t)\right)$, with fixed initial position $x_{k}(0)=y$, and a sequence of time $t_{k}, k=1,2, \ldots$, such that $t_{k} \rightarrow n \pi, x_{k}\left(t_{k}\right) \rightarrow x$ and $\tau\left(t_{k}\right) \rightarrow \infty$, as $k \rightarrow \infty$. Thus $E(t, x, y)$ is singular everywhere when $t=n \pi$, $n=0, \pm 1, \ldots$, as we see explicitly from Mehler's formula (1.8) above. Since the smooth perturbation of $x^{2} / 2$ becomes negligible in the high energy limit of the Hamiltonian flow, this argument also explains the recurrence of singularity results of [9] and [21].

When $V(x)$ is subquadratic, the period of the trajectories diverges to infinity as energy increases to infinity. Hence, the trajectories with $x(0)=y$ and initial momentum $p(0),|p(0)| \rightarrow \infty$, will have gone instantaneously to infinity and will never come back to any point of configuration space. These trajectories produce no singularities anywhere and $E(t, x, y)$ is smooth everywhere. If $V(x)$ is superquadratic, on the other hand, the period of the trajectories decreases to 0 as the energy grows to $\infty$. Hence, for any two spatial points $x, y$ and any time $t$, there are trajectories with arbitrarily high energy that leave $y$ at time zero and reach $x$ at time $t$. Such trajectories create the singularity of the FDS at $(t, x, y)$ and $E(t, x, y)$ is nowhere smooth. Though this heuristic argument is, of course, not a proof of the above theorems, it gives a clear explanation why the drastic change of smoothness of FDS takes place as the potential $V(x)$ changes from sub-quadratic to super-quadratic at infinity. Indeed, the proof of Theorem 1.1 that we shall present in Sect. 2 is based on this semi-classical picture.

We now describe the plan of the paper, introduce some additional notation and then outline the proofs of Theorem 1.1 and Theorem 1.2. In Sect. 2, we prove that $E(t, s, x, y)$ is everywhere smooth with respect to $(x, y)$ if $V(t, x)$ is sub-quadratic. The proof is based on two facts, the first, that Theorem 1.1 holds for small time $|t-s| \leqq T(V)$ and, hence, the propagator $U(t, s)$ is continuous from the Schwartz space $\mathscr{S}\left(\mathbf{R}^{m}\right)$ onto itself for all $t, s \in \mathbf{R}$ (this is due to Fujiwara [6]); the second, that for $|t-s| \leqq T, T$ being arbitrarily large, the Hamiltonian flow of (1.2) has a generating function $S(t, s, x, y)$ outside a bounded set $\left\{(x, y):|x|^{2}+|y|^{2} \geqq C_{T}^{2}\right\}$ and it satisfies the estimate

$$
\left\|\partial_{x}^{2} S(t, s, x, y)-(t-s)^{-1}\right\| \leqq 10^{-10}|t-s|^{-1}
$$

as indicated in statement (1). This is a consequence of the sub-quadratic behavior of the potential. The major part of the proof is devoted to the construction of the generating function and to the study of its properties. For arbitrary $0<t-s \leqq T$, we divide $s=t_{0}<t_{1}<\cdots<t_{N}=t$ such that $\left|t_{j}-t_{j-1}\right| \leqq T(V), j=1, \ldots, N$, and write $U(t, s)=U\left(t_{N}, t_{N-1}\right) \cdots U\left(t_{1}, t_{0}\right)$. We may suppose by induction that $E\left(t_{N-1}, t_{0}, x, y\right)$ satisfies the statements of Theorem 1.1 and write

$$
E\left(t_{N-1}, s, x, y\right)=E^{(1)}\left(t_{N-1}, s, x, y\right)+e^{i S\left(t_{N-1}, s, x, y\right)} a\left(t_{N-1}, s, x, y\right),
$$


where $E^{(1)}$ is smooth with compact support with respect to $(x, y)$ and $a\left(t_{N-1}, s, x, y\right)$ is smooth and is supported in the set $\left\{(x, y) ;|x|^{2}+|y|^{2} \geqq C_{T}^{2}\right\}$. (Here we have absorbed the factor $(2 \pi i(t-s))^{-m / 2}$ into $a$ and we will continue to do so in what follows.) Using the short time result, we write

$$
\begin{aligned}
E(t, s, x, y)= & \int e^{i S\left(t, t_{N-1}, x, z\right)} a\left(t, t_{N-1}, x, z\right) E^{(1)}\left(t_{N-1}, s, z, y\right) d z \\
& +\int e^{i\left(S\left(t, t_{N-1}, x, z\right)+S\left(t_{N-1}, s, z, y\right)\right)} a\left(t, t_{N-1}, x, z\right) a\left(t_{N-1}, s, z, y\right) d z \\
= & E_{1}(t, s, x, y)+E_{2}(t, s, x, y) .
\end{aligned}
$$

Since $E^{(1)}\left(t_{N-1}, s, x, y\right)$ is $C_{0}^{\infty}\left(\mathbf{R}^{2 m}\right)$ with respect to $(x, y)$, the fact that $U\left(t, t_{N-1}\right)$ is a continuous operator in $\mathscr{S}\left(\mathbf{R}^{m}\right)$ implies that $E_{1}(t, s, x, y) \in \mathscr{S}\left(\mathbf{R}_{x}^{m} \times \mathbf{R}_{y}^{m}\right)$. To analyze $E_{2}(t, s, x, y)$ we apply the method of stationary phase using the fact that

$$
\partial_{z}^{2} S\left(t, t_{N-1}, x, z\right)+\partial_{z}^{2} S\left(t_{N-1}, s, z, y\right) \sim\left(t-t_{N-1}\right)^{-1}+\left(t_{N-1}-s\right)^{-1}
$$

is non-singular, which yields the desired properties of $E(t, s, x, y)$. Thus the proof of Theorem 1.1 more or less follows the semi-classical picture outlined in the preceding "explanation" of the theorems.

In Sect. 3, we prove that $E(t, x, y)$ is nowhere in $\mathscr{L}_{1, \text { loc }}^{1 / 2 c}\left(\mathbf{R}^{3}\right)$ when $V(t, x)=V(x)$ is super-quadratic and the spatial dimension $m=1$. In contrast to the proof of Theorem 1.1, the proof here is indirect in the sense that it heavily relies upon the spectral theory of the operator $H=-(1 / 2) d^{2} / d x^{2}+V(x)$. By the definition of Bessel space, it suffices, by the Riemann-Lebesgue theorem, to show that for nonnegative $\rho, \Phi, \Psi \in C_{0}^{\infty}\left(\mathbf{R}^{1}\right)$,

$$
\int_{\mathbf{R}^{3}} E(t, x, y) \rho(t) \Phi(y) \Psi(x) e^{i(t \lambda+y \xi-x \eta)} d t d x d y=\left(\widehat{\rho}(\lambda-H) \Phi(y) e^{i y \cdot \xi}, \Psi(x) e^{i x \cdot \eta}\right),
$$

does not go to zero faster than $C(|\lambda|+|\xi|+|\eta|)^{-1 / 2 c}$ as $|\lambda|+|\xi|+|\eta| \rightarrow \infty$. Here $\widehat{\rho}$ is the Fourier transform of $\rho$,

$$
\widehat{\rho}(\lambda)=\int_{-\infty}^{\infty} e^{-i t \lambda} \rho(t) d t
$$

and $(\cdot, \cdot)$ in $(1.11)$ is the inner product in $L^{2}\left(\mathbf{R}^{1}\right)$. We set $\xi=\eta= \pm \sqrt{2 \lambda}$ in (1.11) and let $\lambda \rightarrow \infty$ along the eigenvalues $\lambda_{n}$ of $H$. Since $V$ is super-quadratic, the one dimensional operator $H$ has only eigenvalues $\lambda_{n}$ tending to $\infty$ and the spacing of neighboring eigenvalues increases algebraically as follows: $\left|\lambda_{n}-\lambda_{n \pm 1}\right| \geqq C \lambda_{n}^{1 / 2-1 / 2 c}$. Hence, modulo $O\left(\lambda_{n}^{-N}\right)$, only the projection to the $n^{\text {th }}$ eigenfunction $u_{n}(x)$ contributes to $(1.11)$,

$$
(1.11)=\widehat{\rho}(0)\left(\Phi(x) e^{i x \cdot \xi}, u_{n}\right)\left(u_{n}, \Psi(x) e^{i x \cdot \eta}\right)+O\left(\lambda_{n}^{-N}\right),
$$

where $N$ is arbitrary large. But, on every compact interval, $u_{n}(x)$ asymptotically approaches a plane wave as $n \rightarrow \infty, u_{n}(x) \sim \operatorname{Re}\left\{C_{\lambda_{n}} e^{i \sqrt{2 \lambda_{n}} x}\right\}, C_{\lambda_{n}}$ being a complex constant satisfying the lower bound $\left|C_{\lambda_{n}}\right| \geqq C \lambda_{n}^{-1 / 4 c}$. Thus, we have $|(1.11)| \geqq$ $C \lambda_{n}^{-1 / 2 c}$ and $E(t, x, y)$ is nowhere in $\mathscr{L}_{1, \text { loc }}^{1 / 2 c}\left(\mathbf{R}^{3}\right)$. In particular, it is nowhere in $C^{1}$.

In what follows various constants whose specific values are not important will be denoted by the same character $C$. These constants may differ from place to place. 


\section{Sub-Quadratic Potentials - Proof of Theorem 1.1}

In this section, we always assume that $V(t, x)$ is subquadratic, viz, that the condition (1.3) is satisfied. Let $V^{\prime}(t, x)$ denote the vector $\partial_{x} V(t, x), V^{\prime \prime}(t, x)$ the matrix $\partial_{x}^{2} V(t, x)$ and set $M=\sup _{t, x}\left\|V^{\prime \prime}(t, x)\right\|$. For $0<\delta<1, L_{\delta}$ denotes the smallest number such that

$$
\sup _{t \in \mathbf{R}}\left\|V^{(j)}(t, x)\right\| \leqq \delta|x|^{2-j}, \quad \text { for }|x| \geqq L_{\delta}, \quad j=0,1,2,
$$

where $\|\cdot\|$ should be understood as the absolute value, the Euclidean norm of a vector, and the matrix norm of a linear operator in $\mathbf{R}^{m}$, for $j=0,1,2$ respectively. Set

$$
M_{j, \delta}=\sup _{t \in \mathbf{R},|x| \leqq L_{\delta}}\left\|V^{(j)}(t, x)\right\| .
$$

In what follows, when there is no confusion, we shall often suppress the explicit dependence of various quantities on the independent variables or parameters.

We begin by studying the trajectories of the Hamilton flow, $(x(t, s, y, k), p(t, s$, $y, k)$ ), corresponding to (1.1), viz, the solutions of (1.2). Standard arguments in ordinary differential equations (see e.g. Coddington-Levinson [3]) show that

- for fixed $(t, s),(x(t, s, y, k), p(t, s, y, k))$ is $C^{\infty}$ with respect to $(y, k)$,

- the derivatives of $(x(t, s, y, k), p(t, s, y, k))$ with respect to $(y, k)$ are $C^{\sigma+1}$ with respect to the all variables $(t, s, y, k)$.

Furthermore $x(t)=x(t, s, y, k)$ satisfies the integral equation

$$
x(t)=y+k(t-s)-\int_{s}^{t}(t-\tau) V^{\prime}(\tau, x(\tau)) d \tau .
$$

Lemma 2.1. Let $N=\max \left(1,2 M, 2 \sup _{t \in \mathbf{R}}|V(t, 0)|\right)$. Then, for any $(y, k) \in \mathbf{R}^{m} \times \mathbf{R}^{m}$ and $(t, s) \in \mathbf{R} \times \mathbf{R}$,

$$
\left(1+|x(t, s, y, k)|^{2}+|p(t, s, y, k)|^{2}\right) \leqq e^{2 N|t-s|}\left(1+|y|^{2}+|k|^{2}\right) .
$$

Proof. Write $F(t)=\left(1+|x(t)|^{2}+|p(t)|^{2}\right)^{1 / 2}$ and denote by the $t$-derivative. From the Schwarz inequality, and the fact that $(x(t), p(t))$ satisfies Eq. (1.2), we have

$$
(d / d t) F(t)^{2} \leqq 2 F(t)\left(|\dot{x}(t)|^{2}+|\dot{p}(t)|^{2}\right)^{1 / 2} \leqq 2 F(t)\left(|p(t)|^{2}+\left|V^{\prime}(t, x(t))\right|^{2}\right)^{1 / 2} .
$$

By the mean value theorem, $\left|V^{\prime}(t, x)\right| \leqq\left|V^{\prime}(t, 0)\right|+M|x|$, which implies

$$
(d / d t) F(t)^{2} \leqq 2 F(t)\left(|p(t)|^{2}+2\left|V^{\prime}(t, 0)\right|^{2}+2 M^{2}|x(t)|^{2}\right)^{1 / 2} \leqq 2 N F(t)^{2} .
$$

The estimate (2.4) now follows by quadrature.

Notational remark. In the following we will use $10^{-10}$ as a generically small constant. This notation has an advantage of indicating the number of estimates needed to arrive at our final result. Thus the estimates in the first stage are proportional to $10^{-10}$, the estimates in the second stage are proportional to $10^{-9}$, etc. 
Lemma 2.2. Let $T>0$ and $C_{1}>0$. Then, there exists $C_{2}>0$ such that the following estimates are satisfied for $|y| \leqq C_{1},|t-s| \leqq T$, and $|k| \geqq C_{2}$ :

$$
\begin{gathered}
|x(t, s, y, k)-y-(t-s) k| \leqq 10^{-10}(1+|t-s||k|) \\
|p(t, s, y, k)-k| \leqq 10^{-10}|k|
\end{gathered}
$$

Proof. We prove (2.5) for the case $s=0 \leqq t \leqq s+T$ only. The proofs for the general case, and for $p(t, s, y, k)$, are similar. Define $f(t)$ by

$$
f(t)=-\int_{0}^{t}(t-s) V^{\prime}(s, y+s k) d s
$$

and let $0<\delta<1$. We split the interval $[0, t]$ into two parts, $I_{1}=\{0 \leqq s \leqq t$ : $\left.|y+s k| \geqq L_{\delta}\right\}$ and $I_{2}=\left\{0 \leqq s \leqq t:|y+s k| \leqq L_{\delta}\right\}$. It follows, by the definition of $L_{\delta}$, that $\left|V^{\prime}(s, y+s k)\right| \leqq \delta|y+s k|$ for $s \in I_{1}$ and that $\left|V^{\prime}(s, y+s k)\right| \leqq M_{1, \delta}$ for $s \in I_{2}$. Since the measure of $I_{2}$ does not exceed $2 L_{\delta} /|k|$,

$$
\begin{aligned}
|f(t)| & \leqq|t| \int_{0}^{t}\left|V^{\prime}(s, y+s k)\right| d s \leqq|t|\left(2 L_{\delta} /|k|\right) M_{1, \delta}+\delta \int_{0}^{t}|y+s k| d s \\
& \leqq|t|\left\{\left(2 L_{\delta} /|k|\right) M_{1, \delta}+\delta|y|\right\}+\delta|t|^{2}|k|
\end{aligned}
$$

We denote $g(t)=x(t)-y-t k$ and write, via the mean value theorem, the integral Eq. (2.3) in the form

$$
g(t)=f(t)-\int_{0}^{t}(t-s)\left\{\int_{0}^{1} V^{\prime \prime}(s, y+k s+\theta g(s)) d \theta\right\} g(s) d s
$$

Using the estimate $\left\|V^{\prime \prime}(s, \ldots)\right\| \leqq M$ and applying Gronwall's inequality, we obtain

$$
\begin{aligned}
|g(t)| & \leqq|f(t)|+\int_{0}^{t}(t-s) M|g(s)| d s \leqq|f(t)|+T M \int_{0}^{t}|g(s)| d s \\
& \leqq|f(t)|+M T \int_{0}^{t} e^{M T(t-s)}|f(s)| d s \leqq \max _{0 \leqq s \leqq t}|f(s)| \cdot e^{M t T}
\end{aligned}
$$

Inserting (2.7) into (2.9) yields, for $0 \leqq t \leqq T,|y| \leqq C_{1}$, and $|k| \geqq C_{2}$,

$$
|g(t)| \leqq T e^{M T^{2}}\left\{\left(2 L_{\delta} / C_{2}\right) M_{1, \delta}+\delta C_{1}\right\}+|t k|\left(T e^{M T^{2}} \delta\right) .
$$

Thus, choosing $\delta>0$ sufficiently small and $C_{2}$ large enough, we arrive at the estimate (2.5).

Corollary 2.3. Let $T>0$. Then, there exists a constant $C_{3}$ such that, for any $R \geqq 1$, the following estimate holds for $(t, s, y, k)$ satisfying $|t-s| \leqq T$ and $y^{2}+$ $(t-s)^{2} k^{2} \leqq R^{2}$ :

$$
|x(t, s, y, k)-y-(t-s) k| \leqq C_{3} R
$$


Proof. We assume $s=0 \leqq t \leqq s+T$ and use the same notation as in the proof of the previous lemma. Using the bound $\left|V^{\prime}(t, x)\right| \leqq C(1+|x|)$, we estimate

$$
|f(t)| \leqq t \int_{0}^{t}\left|V^{\prime}(s, y+s k)\right| d s \leqq t^{2} C(1+|y|+t|k|),
$$

and insert the latter estimate into (2.9). The result is

$$
|g(t)| \leqq e^{M t T} t^{2} C(1+|y|+t|k|) .
$$

If we take $C_{3}=2 \sqrt{2} C T^{2} e^{M T^{2}}$, this clearly produces $(2.11)$ for $R \geqq 1$.

The following lemma, which is the key lemma of this section, demonstrates that, in the sub-quadratic potential field, the variations along a trajectory remain almost constant for a finite interval of time, if the trajectory starts either with a large initial momentum, $k$, or from a point, $y$, far away from the center of the potential. The proof will exhibit why this happens: if $|y|$ is large while $|k|$ remains small, the trajectory remains in the region where $\left\|V^{\prime \prime}(t, x)\right\|$ is small; if $|k|$ is large, on the other hand, though the trajectory can enter the region where $\left\|V^{\prime \prime}(t, x)\right\|$ is large, the sojourn time in that region is short because the velocity is high and because re-entrance to the region is forbidden, due to the long period of the trajectory.

We now state the lemma and give a formal proof. We use the notation $\partial_{y} x(t, s, y, k)$, etc. to represent the differential of the map of $y \rightarrow x(t, s, y, k)$, etc.

Lemma 2.4. Let $T>0$. Then, there is a constant $R \geqq 0$ such that the following estimates hold for $(t, s, y, k)$ satisfying $|t-s| \leqq T$ and $|y|^{2}+|k|^{2} \geqq R^{2}$ :

$$
\begin{gathered}
\left\|\partial_{y} x(t, s, y, k)-1\right\| \leqq 10^{-10} . \\
\left\|\partial_{k} x(t, s, y, k)-(t-s)\right\| \leqq 10^{-10}|t-s| . \\
\left\|\partial_{y} p(t, s, y, k)\right\|+\left\|\partial_{k} p(t, s, y, k)-1\right\| \leqq 10^{-10} .
\end{gathered}
$$

Here 1 (resp. $(t-s)$ ) on the left-hand sides stands for the $m \times m$ identity matrix (resp. $(t-s)$ times the identity matrix).

Proof. We only prove (2.12) for $s=0$ and $0 \leqq t \leqq T$. Proofs for other cases are similar. By differentiating (2.3) with respect to $y$, we have an equation for the matrix-valued function $\partial_{y} x(t)$,

$$
\partial_{y} x(t)=1-\int_{0}^{t}(t-s) V^{\prime \prime}(s, x(s)) \partial_{y} x(s) d s .
$$

Since $\left\|(t-s) V^{\prime \prime}(s, x)\right\| \leqq T M$, Gronwall's inequality implies

$$
\left\|\partial_{y} x(t)\right\| \leqq e^{M T t}, \quad 0 \leqq t \leqq T,
$$

which, when applied to $(2.15)$, in turn produces the estimate

$$
\left\|\partial_{y} x(t)-1\right\| \leqq T e^{M T^{2}} \int_{0}^{T}\left\|V^{\prime \prime}(s, x(s))\right\| d s .
$$


Given $T$, we set $\delta=10^{-10}\left(T^{2} e^{M T^{2}}\right)^{-1} / 2$. We let $C_{2}$ be the constant in Lemma 2.2 corresponding to $C_{1}=L_{\delta}$, where $L_{\delta}$ is defined by (2.1). By replacing $C_{2}$ by a larger constant if necessary, we may assume $C_{2}>4 \cdot 10^{10} T e^{M T^{2}}\left(2 L_{\delta}+1\right)$. We show that (2.12) is satisfied if we take $R=e^{N T}\left(1+C_{2}+L_{\delta}\right)$, where $N$ is the constant of Lemma 2.1.

We decompose the interval $[0, T]$ into two subsets

$$
I_{1}=\left\{0 \leqq t \leqq T:|x(t)| \geqq L_{\delta}\right\}, \quad I_{2}=\left\{0 \leqq t \leqq T:|x(t)| \leqq L_{\delta}\right\} .
$$

Since $\left\|V^{\prime \prime}(s, x(s))\right\| \leqq \delta$ for $s \in I_{1}$, by definition,

$$
T e^{M T^{2}} \int_{I_{1}}\left\|V^{\prime \prime}(s, x(s))\right\| d s \leqq T^{2} e^{M T^{2}} \delta=10^{-10} / 2,
$$

and (2.12) follows if $I_{2}=\emptyset$. Suppose, therefore, that $I_{2} \neq \emptyset$ and $\left|x\left(t_{0}\right)\right| \leqq L_{\delta}$ for some $t_{0} \in[0, T]$. We have $\left|p\left(t_{0}\right)\right| \geqq C_{2}$; otherwise by Lemma 2.1,

$$
\begin{aligned}
R^{2}+1 & \leqq|k|^{2}+|y|^{2}+1 \leqq e^{2 N T}\left(1+\left|p\left(t_{0}\right)\right|^{2}+\left|x\left(t_{0}\right)\right|^{2}\right) \\
& \leqq e^{2 N T}\left(1+C_{2}+L_{\delta}\right)^{2}=R^{2},
\end{aligned}
$$

which is a contradiction. It follows, by virtue of Lemma 2.2, that

$$
\left|x(t)-x\left(t_{0}\right)-\left(t-t_{0}\right) p\left(t_{0}\right)\right| \leqq 10^{-10}\left(1+\left|\left(t-t_{0}\right) p\left(t_{0}\right)\right|\right)
$$

and, if $T \geqq\left|t-t_{0}\right| \geqq 2\left(2 L_{\delta}+1\right) / C_{2}$, then $|x(t)| \geqq\left(1-10^{-10}\right)\left|t-t_{0}\right|\left|p\left(t_{0}\right)\right|-$ $\left|x\left(t_{0}\right)\right|-10^{-10}>L_{\delta}$. This implies that the measure of $I_{2}$ does not exceed $2\left(2 L_{\delta}+\right.$ 1) $/ C_{2}$, and

$$
T e^{M T^{2}} \int_{I_{2}}\left\|V^{\prime \prime}(s, x(s))\right\| d s \leqq T e^{M T^{2}} M\left|I_{2}\right| \leqq 10^{-10} / 2,
$$

which concludes the proof of the lemma.

The following estimations of the higher derivatives of $x(t, s, y, k)$ and $p(t, s, y, k)$ are well known ([5], Proposition 1.4).

Lemma 2.5. For $|\alpha+\beta| \geqq 2$ there exists a constant $C_{\alpha \beta}$ such that for all $(t, s, x, y)$,

$$
\begin{aligned}
& \left|\partial_{y}^{\alpha} \partial_{k}^{\beta} x(t, s, y, k)\right| \leqq C_{\alpha \beta}|t-s|^{2+|\beta|}, \\
& \left|\partial_{y}^{\alpha} \partial_{k}^{\beta} p(t, s, y, k)\right| \leqq C_{\alpha \beta}|t-s|^{1+|\beta|} .
\end{aligned}
$$

We now set $\tilde{x}(t, s, y, k)=x\left(t, s, y,(t-s)^{-1} k\right)$ (cf. [5]) and consider the two parameter family of mappings $\Gamma(t, s)$ in $\mathbf{R}^{m} \times \mathbf{R}^{m}$ given by

$$
\Gamma(t, s)(y, k)=(y, \tilde{x}(t, s, y, k)), \quad(y, k) \in \mathbf{R}^{m} \times \mathbf{R}^{m} .
$$

Lemma 2.4 and Lemma 2.5 imply that all the derivatives of the mapping $\Gamma(t, s)(y, k)$ are bounded uniformly for $0<|t-s| \leqq T$ and that the differential at $(y, k)$, which we denote by $J(t, s, y, k)$, satisfies, for all $|y|^{2}+|k|^{2} \geqq R^{2}$ and $0<|t-s| \leqq T$,

$$
\|J(t, s, y, k)-A\| \leqq 10^{-10}, \quad A=\left(\begin{array}{cc}
1 & 1 \\
0 & 1
\end{array}\right),
$$


where 1 stands for the $m \times m$ identity matrix and $R$ is $T+1$ times the constant $R$ appeared in Lemma 2.4. The matrix $A$ is non-singular and $\sqrt{2} \leqq\|A\|=\left\|A^{-1}\right\| \leqq$ $\sqrt{3}$ as a linear operator on $\mathbf{R}^{m} \times \mathbf{R}^{m}$.

The following lemma shows that such maps $\Gamma(t, s)$ are diffeomorphisms on the exterior of a ball, and estimates the size of the image. We denote

$$
B_{\geqq R}=\left\{z \in \mathbf{R}^{\ell}:|z| \geqq R\right\}, \quad B_{\leqq R}=\left\{z \in \mathbf{R}^{\ell}:|z| \leqq R\right\},
$$

etc. We remark that, when $T>0$ is small, (2.20) holds with $R=0$ because the right-hand side of (2.16) clearly converges to zero as $T \rightarrow 0$ (cf. Fujiwara [5]).

Lemma 2.6. Let $A$ be an $\ell \times \ell$ non-singular matrix, $F: \mathbf{R}^{\ell} \rightarrow \mathbf{R}^{\ell}$ be $C^{\infty}$ with $\left|\partial^{\alpha} F(z)\right| \leqq C_{\alpha}$, for $|\alpha| \geqq 1$, and let $R \geqq 0$. Suppose that the differential $\partial F(z)$ of $F(z)$ satisfies

$$
\|\partial F(z)-A\|<\left(\pi\left\|A^{-1}\right\|\right)^{-1}, \quad \text { for } z \in B_{\geqq R} .
$$

Set $C_{4}=56\left(1+\pi\|A\|\left\|A^{-1}\right\|\right), C_{5}=2\left(\|A\|+\left(\pi\left\|A^{-1}\right\|\right)^{-1}\right)$ and $K=\sup _{|z|=R} \mid F(z)$ $-A z \mid$. Then:

1. The map $F$ is a diffeomorphism from $B_{\geqq R}$ to its image.

2. The image, $F\left(B_{\geqq R}\right)$, contains the exterior of the ball $B_{\geqq}\left(C_{4} K+C_{5} R\right)$.

3. When $|z| \geqq \max \left\{6 K\left\|A^{-1}\right\|, R\right\}, F(z)$ satisfies $|F(z)| \geqq|z| /\left(2\left\|A^{-1}\right\|\right)$. In particular, we have an upper bound of the image of the exterior of balls,

$$
F\left(B_{\geqq r_{1}}\right) \subset B_{\geqq r_{1} /\left(2\left\|A^{-1}\right\|\right)}, \quad \text { for } r_{1} \geqq \max \left\{6 K\left\|A^{-1}\right\|, R\right\} .
$$

Proof. The inequality (2.21) implies $\left\|A^{-1} \partial F(z)-1\right\|<\pi^{-1}$. Hence, $\partial F(z)$ is nonsingular and $F$ is locally diffeomorphic on $B \geqq R$. Denote $\kappa=\left(\pi\left\|A^{-1}\right\|\right)^{-1}$. If $\{\gamma(s)$ : $0 \leqq s \leqq 1\}$ is a rectifiable curve in $B \geqq R$ of length $\|\gamma\|$ connecting two points $z, w \in$ $B_{\geqq R}$, we have

$$
|F(z)-F(w)-A(z-w)|=\left|\int_{0}^{1}(\partial F(\gamma(s))-A) \dot{\gamma}(s) d s\right| \leqq \kappa\|\gamma\|,
$$

where equality holds only if $\|\gamma\|=0$. Connecting $z$ and $w$ in $B \geqq R$ by a half circle with these points at the ends, we deduce from (2.22) that

$$
|F(z)-F(w)-A(z-w)| \leqq \kappa \pi|z-w|=\left\|A^{-1}\right\|^{-1}|z-w|,
$$

where again equality holds only when $z=w$. Hence $F$ is one to one on $B \geqq R$ and $F$ is diffeomorphic there. This proves the first statement. deduce

Taking the line segment connecting $z$ and $z_{0}=R z /|z|$ as $\gamma(s)$ in (2.22), we

$$
|F(z)-A z| \leqq\left|F\left(z_{0}\right)-A z_{0}\right|+\kappa\left|z-z_{0}\right| \leqq K+\kappa|z| .
$$

It follows that if $|z| \geqq \max \left\{6 K\left\|A^{-1}\right\|, R\right\}$,

$$
|F(z)| \geqq\left\|A^{-1}\right\|^{-1}|z|-K-\kappa|z| \geqq\left(2\left\|A^{-1}\right\|\right)^{-1}|z|,
$$

which proves the third statement.

To prove the second statement, we modify $F(z)$ as follows and apply Hadamard's global inverse mapping theorem. Take $\phi \in C_{0}^{\infty}(\mathbf{R})$ such that $0 \leqq \phi(t) \leqq 1, \phi(t)=1$ 
for $|t| \geqq 1, \phi(t)=0$ for $|t| \leqq 1 / 2$, and $\left|\phi^{\prime}(t)\right| \leqq 21 / 10$. For $0<\delta<3 / 2$ we define, $F_{\delta}(z)$ by

$$
F_{\delta}(z)= \begin{cases}F(z), & |z| \geqq 3 R / \delta, \\ \phi(\delta|z| / 3 R) F(z)+(1-\phi(\delta|z| / 3 R)) A z, & 3 R / 2 \delta \leqq|z| \leqq 3 R / \delta \\ A z, & |z| \leqq 3 R / 2 \delta\end{cases}
$$

The map $F_{\delta}(z)$ is obviously $C^{\infty}$ and satisfies $\left|\partial^{\alpha} F_{\delta}(z)\right| \leqq C_{\alpha \delta}$. A short computation shows that, for $3 R / 2 \delta \leqq|z| \leqq 3 R / \delta$,

$$
\left\|\partial F_{\delta}(z)-A\right\| \leqq(\delta / 3 R)\left|\phi^{\prime}(\delta|z| / 3 R)\right||F(z)-A z|+\kappa .
$$

Since $\left|\phi^{\prime}\right| \leqq 21 / 10$, the inequality (2.23) now implies the estimate

$$
\left\|\partial F_{\delta}(z)-A\right\| \leqq(21 \delta / 30 R)(K+\kappa(3 R / \delta))+\kappa=(21 / 30 R) K \delta+(31 / 10) \kappa,
$$

which is $\leqq\left(1-10^{-10}\right)\left\|A^{-1}\right\|^{-1}$ provided $\delta \leqq(R / 18 K) \kappa$. Thus, if we set $\delta=$ $\min (1,(R / 18 K) \kappa), F_{\delta}(z)$ is a global diffeomorphism of $\mathbf{R}^{m}$ which is identical with $F(z)$ on $B \geqq 3 R / \delta$. It follows that

$$
F\left(B_{\geqq R}\right) \supset F_{\delta}\left(B_{\geqq 3 R / \delta}\right)=\mathbf{R}^{m} \backslash F_{\delta}\left(B_{\leqq 3 R / \delta}\right) .
$$

Since (2.23) implies that, for $|z| \leqq 3 R / \delta$,

$$
\begin{aligned}
|F(z)| & \leqq|A z|+K+\kappa|z| \leqq(\|A\|+\kappa)(3 R / \delta)+K \\
& \leqq \max \left(2 R(\|A\|+\kappa)+K, 56\left(1+\pi\left\|A^{-1}\right\|\|A\|\right) K\right) \leqq C_{4} K+C_{5} R,
\end{aligned}
$$

statement (2) now follows.

In the remainder of this section, we fix $T>0$ arbitrarily large, and then, take $R>1$ in such a way that (2.20) is satisfied for all $(t, s, y, k)$ satisfying $|y|^{2}+|k|^{2} \geqq R^{2}$ and $0<|t-s| \leqq T$. We apply Lemma 2.6 to the triplet $(A, F, R)$ consisting of the nonsingular matrix $A=\left(\begin{array}{ll}1 & 1 \\ 0 & 1\end{array}\right)$ the map $F=\Gamma(t, s)$ and this constant $R$. Recall that $\sqrt{2} \leqq\|A\|=\left\|A^{-1}\right\| \leqq \sqrt{3}$. We let $C_{3}$ be the constant in Corollary 2.3 and set, as in the previous lemma,

$$
\begin{gathered}
C_{4}=56\left(1+\pi\|A\|\left\|A^{-1}\right\|\right)(<600), \quad C_{5}=2\left(\|A\|+\pi^{-1}\left\|A^{-1}\right\|^{-1}\right)(<4), \\
C_{6}=C_{3} C_{4}+C_{5}, \quad \text { and } \quad R_{0}=\left\{6 \sqrt{3}\left(C_{3}+1\right)+T\right\} R .
\end{gathered}
$$

The constant $K(t, s)$, which is the $K$ of the previous lemma for this triplet $A, \Gamma(t, s)$ and $R$, satisfies, by virtue of Corollary 2.3,

$$
K(t, s)=\sup _{|y|^{2}+|(t-s) k|^{2}=R^{2}}|x(t, s, y, k)-y-(t-s) k| \leqq C_{3} R,
$$

and hence $\max \left\{6 K(t, s)\left\|A^{-1}\right\|, R\right\} \leqq R_{0}$. Thus, Lemma 2.6 implies the first part and the relation (2.24) of the following lemma.

Lemma 2.7. Let $R \geqq 1$ and $R_{0}$ be as above. Then, for $0<|t-s| \leqq T$, the map $\Gamma(t, s)$ is diffeomorphic from $B_{\geqq R}$ to its image. Moreover, if $r \geqq R_{0}$,

$$
B_{\geqq} C_{6} r \subset(t, s)\left(B_{\geqq r}\right) \subset B_{\geqq r / 2 \sqrt{2}},
$$

and the inverse image in $\mathbf{R}^{m} \times \mathbf{R}^{m}$ of $(x, y) \in \Gamma(t, s)\left(B_{\geqq r}\right)$ is unique. 
Proof. We have only to prove the last statement. But, this is obvious because of the following two facts: firstly, Corollary 2.3 and (2.24) imply, for $r \geqq R_{0}$,

$$
\Gamma(t, s)\left(B_{\leqq}\right) \cap \Gamma(t, s)\left(B_{\geqq r}\right) \subset B_{\leqq}\left(C_{3}+3\right) R \cap B_{\geqq R_{0} / 2 \sqrt{2}}=\emptyset,
$$

and secondly, $\Gamma(t, s)$ is diffeomorphic on $B_{\geqq R}$.

We set $C_{T}=C_{6} R_{0}$ and write, as in Theorem 1.1,

$$
\Omega_{T}=\left\{(t, s, x, y) \in \mathbf{R}^{2 m+2}:|t-s| \leqq T,|x|^{2}+|y|^{2} \geqq C_{T}^{2}\right\} .
$$

Lemma 2.7 and the definition of $\Gamma(t, s)$ imply that, for any $(t, s, x, y) \in \Omega_{T}$, there is a unique $k=k(t, s, x, y) \in \mathbf{R}^{m}$ such that $x=x(t, s, y, k)$, and this $k$ satisfies $y^{2}+$ $(t-s)^{2} k^{2} \geqq R_{0}^{2}$; and hence, $y^{2}+k^{2} \geqq R^{2}$. Setting $k=k(t, s, x, y)$, we define the function $S(t, s, x, y)$ on $\Omega_{T}$ by

$$
S(t, s, x, y)=\int_{s}^{t}\left(p(\tau, s, y, k)^{2} / 2-V(\tau, x(\tau, s, y, k))\right) d \tau .
$$

It is well known (cf. e.g. [2]) that $S=S(t, s, x, y)$ is a generating function of the Hamilton flow defined by (1.2),

$$
\begin{gathered}
\left(\partial_{x} S\right)(t, s, x(t, s, y, k), y)=p(t, s, y, k), \\
\left(\partial_{y} S\right)(t, s, x(t, s, y, k), y)=-k,
\end{gathered}
$$

and $S$ satisfies the Hamilton-Jacobi equation corresponding to (1.1),

$$
\partial_{t} S+\left(\partial_{x} S\right)^{2} / 2+V(t, x)=0, \quad \partial_{s} S-\left(\partial_{y} S\right)^{2} / 2-V(s, y)=0 .
$$

The latter property, however, will not be used explicitly in this paper.

Lemma 2.8. On the domain $\Omega_{T}, S(t, s, x, y)$ is $C^{\infty}$ with respect to $x$ and $y$. All derivatives $\partial_{x}^{\alpha} \partial_{y}^{\beta} S(t, s, x, y)$ are $C^{\sigma+1}$ with respect to $(t, s, x, y)$ and satisfy the following estimates:

$$
\begin{gathered}
\sup _{|\alpha+\beta|=2}\left\|\partial_{x}^{\alpha} \partial_{y}^{\beta}\left\{S(t, s, x, y)-(x-y)^{2} / 2(t-s)\right\}\right\| \leqq 10^{-8}|t-s|^{-1}, \\
\left|\partial_{x}^{\alpha} \partial_{y}^{\beta} S(t, s, x, y)\right| \leqq C_{\alpha \beta}, \quad|\alpha+\beta| \geqq 3 .
\end{gathered}
$$

Proof. The smoothness property of $S(t, s, x, y)$ follows from the definition (2.25) and the corresponding properties of $(x(t, s, y, k), p(t, s, y, k))$ stated in the second paragraph of this section. Differentiation of (2.26) with respect to $k$ gives for $x=$ $x(t, s, y, k)$,

$$
\partial_{x}^{2} S(t, s, x, y)=\left(\partial_{k} p\right)(t, s, y, k) \cdot\left(\partial_{k} x\right)^{-1}(t, s, y, k), \quad y^{2}+k^{2} \geqq R^{2},
$$

where $\left(\partial_{k} x\right)^{-1}$ is the inverse matrix of $\left(\partial_{k} x\right)$. The relation $(2.14), \|\left(\partial_{k} p\right)(t, s, y, k)$ $-1 \| \leqq 10^{-10}$, and the estimate $\left\|\left(\partial_{k} x\right)^{-1}(t, s, y, k)-(t-s)^{-1}\right\| \leqq 10^{-9}|t-s|^{-1}$, which follows readily from (2.13), then yield the estimate (2.28) for the case $|\alpha|=2$. Other cases of (2.28) may be proved similarly by differentiating (2.27) by $k$ and $y$ and by applying Lemma 2.4. Estimates (2.29) may be proved by further differentiating the expressions thus obtained, say (2.30), and then applying Lemma 2.5. We omit the details. 
Because of the remark preceding Lemma 2.6, when $0<|t-s| \leqq T(V), \Gamma(t, s)$ is a global diffeomorphism of $\mathbf{R}^{m} \times \mathbf{R}^{m}$ and $S(t, s, x, y)$ is defined for all $(x, y) \in$ $\mathbf{R}^{m} \times \mathbf{R}^{m}$. It is easy to see (cf. [5]) that (2.26)-(2.29) hold for all $(x, y) \in \mathbf{R}^{2 m}$ when $0<|t-s| \leqq T(V)$.

Proof of Theorem 1.1. We prove the differentiability properties of $\partial_{x}^{\alpha} \partial_{y}^{\beta} E(t, s, x, y)$ with respect to $(t, s, x, y)$ at the end and proceed ignoring this issue for the moment. Fujiwara's theorem [6] shows that Theorem 1.1 holds with $C_{T}=0$ when $T=T(V)$ is sufficiently small. We fix such $T(V)$ and assume, without loss of generality, that (2.28) is satisfied for all $x, y \in \mathbf{R}^{m}$ when $0<|t-s| \leqq T(V)$. We hereafter assume $T>T(V)$ and divide $s=t_{0}<t_{1}<\cdots<t_{N}=s+T$ in such a way that $T(V) / 2 \leqq\left|t_{j}-t_{j-1}\right| \leqq 3 T(V) / 4, j=1, \ldots, N$. By the induction argument and Fujiwara's short time result mentioned above, it suffices to show that Theorem 1.1 holds for $E(t, s, x, y)$ when $t$ runs over $t_{j-1} \leqq t \leqq t_{j}$ under the assumption that Theorem 1.1 is true for $E(t, s, x, y)$ when $s \leqq t \leqq t_{j-1}, j \geqq 2$. We set $r=t_{j-1}-T(V) / 4$. Then $T(V) / 4 \leqq t-r \leqq T(V)$ for $t_{j-1} \leqq t \leqq t_{j}$, and $E(t, r, x, y)$ satisfies the short time results with the smooth bounded amplitude function $a(t, r, x, y)$.

Recall that $C_{T}=C_{6} R_{0}$. Take a $C^{\infty}$ function $\Phi(x, y)$ such that $\Phi(x, y)=0$ for $x^{2}+y^{2} \leqq\left(3 C_{T} / 2\right)^{2}$ and $\Phi(x, y)=1$ for $x^{2}+y^{2} \geqq\left(2 C_{T}\right)^{2}$, and decompose

$$
\begin{aligned}
E(r, s, x, y) & =(1-\Phi(x, y)) E(r, s, x, y)+\Phi(x, y) E(r, s, x, y) \\
& \equiv E^{(1)}(r, s, x, y)+E^{(2)}(r, s, x, y) .
\end{aligned}
$$

Denote by $V^{(j)}(r, s), j=1,2$, the integral operator with the kernel $E^{(j)}(r, s, x, y)$. We have, of course, $U(r, s)=V^{(1)}(r, s)+V^{(2)}(r, s)$ and, because $U(t, s)=U(t, r) U(r, s)$,

$$
U(t, s)=U(t, r)\left(V^{(1)}(r, s)+V^{(2)}(r, s)\right) .
$$

Recall that $U(t, s)$ is a family of continuous operators in $\mathscr{S}\left(\mathbf{R}^{m}\right)$ which is strongly continuous in the parameters $(t, s)$ (we abbreviate such a family as a $C^{0}$-family in the sequel). The function $E^{(1)}(r, s, x, y)$ is $C_{0}^{\infty}$ with respect to $(x, y)$ and continuous with respect to $(r, s, x, y)$ by the induction hypothesis. Hence, $V^{(1)}(r, s)$ is a $C^{0}$ family of continuous operators from $\mathscr{S}^{\prime}\left(\mathbf{R}^{m}\right)$ to $\mathscr{S}\left(\mathbf{R}^{m}\right)$. Thus $U(t, r) V^{(1)}(r, s)$ also constitutes a $C^{0}$-family of continuous operators from $\mathscr{S}^{\prime}\left(\mathbf{R}^{m}\right)$ to $\mathscr{S}\left(\mathbf{R}^{m}\right)$. Thus, by the Schwartz kernel theorem, the operator has the integral kernel $E_{1}(t, s, x, y)=$ $\left\langle U(t, r) V^{(1)}(r, s) \delta_{y}, \delta_{x}\right\rangle$ which belongs to $\mathscr{S}\left(\mathbf{R}^{m} \times \mathbf{R}^{m}\right)$ with respect to $(x, y)$ and the derivatives with respect to $(x, y)$ are continuous with respect to $(t, s, x, y)$, where $\delta$ denotes Dirac's measure. Clearly the function $E_{1}$ may be written in the form required by Theorem 1.1 on the domain $\Omega_{T}$, and we have only to deal with the integral kernel of $U(t, r) V^{(2)}(r, s)$.

By the induction hypothesis and the short time result, the integral kernel $E_{2}(t, s, x, y)$ in question can be written in the form of an oscillatory integral

$$
E_{2}(t, s, x, y)=\int_{\mathbf{R}^{m}} e^{i(S(t, r, x, z)+S(r, s, z, y))} \Phi(z, y) a(t, r, x, z) a(r, s, z, y) d z
$$

Write $\phi(x, z, y)=S(t, r, x, z)+S(r, s, z, y)$ and $A(x, z, y)=\Phi(z, y) a(t, r, x, z) a(r, s, z, y)$. The phase function $\phi(x, z, y)$ is defined on $\left\{(x, z, y): y^{2}+z^{2} \geqq C_{T}^{2}\right\}$ and Lemma 2.8 implies

$$
\left|\partial_{x}^{\alpha} \partial_{y}^{\beta} \partial_{z}^{\gamma} \phi(x, z, y)\right| \leqq C_{\alpha \beta \gamma}, \quad|\alpha|+|\beta|+|\gamma| \geqq 2,
$$


and

$$
\left\|\partial_{z}^{2} \phi(x, z, y)-(t-r)^{-1}-(r-s)^{-1}\right\| \leqq 10^{-8}\left(|t-r|^{-1}+|r-s|^{-1}\right) .
$$

The amplitude function $A(x, z, y)$ is smooth with respect to $x$ and $y$ and the derivatives are continuous with respect to $(t, s, x, z, y)$ satisfying

$$
\left|\partial_{x}^{\alpha} \partial_{y}^{\beta} \partial_{z}^{\gamma} A(x, z, y)\right| \leqq C_{\alpha \beta \gamma}, \quad \text { for all }(\alpha, \beta, \gamma) \text {. }
$$

We first prove that $E_{2}(t, s, x, y)$ is a smooth function of $(x, y)$ by using integration by parts.

Lemma 2.9. $E_{2}(t, s, x, y)$ is $C^{\infty}$ with respect to $(x, y) \in \mathbf{R}^{m} \times \mathbf{R}^{m}$ and the derivatives with respect to $(x, y)$ are continuous with respect to $(t, s, x, y)$.

Proof. We let $K$ be a compact set and prove the lemma for $(x, y) \in K$. We apply Lemma 2.6 to the triplet consisting of the nonsingular matrix $(t-r)^{-1}+(r-s)^{-1}$, the map $z \rightarrow \partial_{z} \phi(x, z, y)$, and the constant $R=C_{T}$. Because of (2.35), the third statement of Lemma 2.6 yields a lower bound for $\left|\partial_{z} \phi(x, z, y)\right|$ for $(x, y) \in K$,

$$
\left|\partial_{z} \phi(x, z, y)\right| \geqq C_{1, K}|z|, \quad|z| \geqq C_{2, K} .
$$

On the other hand (2.34) gives the upper bound,

$$
\left|\partial_{x} \phi(x, z, y)\right|+\left|\partial_{y} \phi(x, z, y)\right|+\left|\partial_{z} \phi(x, z, y)\right| \leqq C(1+|z|), \quad(x, y) \in K .
$$

Define a first order differential operator $L$ by

$$
L=\left(1+\left|\partial_{z} \phi\right|^{2}\right)^{-1}\left(1-i\left(\partial_{z} \phi\right) \cdot \partial_{z}\right) .
$$

The function $e^{i \phi}$ is an eigenfunction of $L$ on the support of $A, L e^{i \phi}=e^{i \phi}$, and integration by parts in the oscillatory integral (2.33) à la Asada-Fujiwara [1] yields

$$
E_{2}(t, s, x, y)=\int\left(L^{\ell} e^{i \phi(x, z, y)}\right) \cdot A(x, z, y) d z=\int e^{i \phi(x, z, y)} \cdot\left({ }^{t} L\right)^{\ell} A(x, z, y) d z,
$$

where ${ }^{t} L$ is the formal transpose of $L$. The lower bound (2.37) and the upper bounds (2.34) and (2.38) of the derivatives of $\phi(x, z, y)$, and the bounds (2.36) on the derivatives of $A$ produce the upper bound for $(x, y) \in K$,

$$
\left|\partial_{x}^{\alpha} \partial_{y}^{\beta}\left\{e^{i \phi(x, z, y)}\left({ }^{t} L\right)^{\ell} A(x, z, y)\right\}\right| \leqq C_{\alpha \gamma}(1+|z|)^{-\ell+|\alpha|+|\beta|} .
$$

Hence, differentiating $(2.40)$ by $(x, y)$ under the integral sign, we conclude that $E_{2}(t, s, x, y)$ is $C^{\infty}$ with respect to $(x, y)$ and $\partial_{x}^{\alpha} \partial_{y}^{\beta} E_{2}(t, s, x, y)$ are continuous with respect to $(t, s, x, y)$.

Next we prove that on $\Omega_{T}$, the derivatives $\partial_{x}^{\alpha} \partial_{y}^{\beta} a_{2}(t, s, x, y)$ of the function $a_{2}$ defined by

$$
a_{2}(t, s, x, y) \equiv e^{-i S(t, s, x, y)} E_{2}(t, s, x, y), \quad(t, s, x, y) \in \Omega_{T},
$$

are uniformly bounded. We apply the method of stationary phase as presented in Lemma 3.2 of Asada-Fujiwara [1]. Note that we only have to show (2.41) for $x^{2}+y^{2} \geqq C_{7}^{2}$, where

$$
C_{7}=20 \sqrt{2} C_{6}^{2} C_{T},
$$

since we already know that on $\Omega_{T}, a_{2}$ is smooth with respect to $(x, y)$ with derivatives which are continuous with respect to all the variables. 
Lemma 2.10. For any $(x, y)$ with $x^{2}+y^{2} \geqq C_{7}^{2}$, there exists a unique point $z=$ $z(x, y)$ of stationary phase for the map $z \rightarrow \phi(x, z, y)$,

$$
\partial_{z} \phi(x, z, y)=0 \text {, }
$$

such that $z^{2}+y^{2} \geqq C_{T}$. Moreover, $z(x, y)$ satisfies $z(x, y)^{2}+y^{2} \geqq\left(2 C_{T}\right)^{2}$, and

$$
\begin{gathered}
\phi(x, z(x, y), y)=S(t, s, x, y), \\
\left|\partial_{x}^{\alpha} \partial_{y}^{\beta} z(x, y)\right| \leqq C_{\alpha \beta}, \quad \text { for all }|\alpha|+|\beta| \geqq 1 .
\end{gathered}
$$

Proof. Recalling Lemma 2.7, we write $x$ in the form $x=x(t, s, y, k)$ for a unique $k \in \mathbf{R}^{m}$. We have, by virtue of (2.26) and (2.27),

$$
\begin{gathered}
\left.\left(\partial_{z} S\right)(t, r, x, z)\right|_{z=x(r, s, y, k)}=-p(r, s, y, k), \\
\left.\left(\partial_{z} S\right)(r, s, z, y)\right|_{z=x(t, s, y, k)}=p(r, s, y, k) .
\end{gathered}
$$

This shows that $z=x(r, s, y, k)(\equiv z(x, y))$ satisfies Eq. (2.42). We show that $z(x, y)^{2}$ $+y^{2} \geqq\left(2 C_{T}\right)^{2}$. The first inclusion relation of Lemma 2.7 and $x^{2}+y^{2} \geqq C_{7}^{2}$ imply

$$
y^{2}+(t-s)^{2} k^{2} \geqq\left(C_{7} / C_{6}\right)^{2}=\left(20 \sqrt{2} C_{T}\right)^{2} .
$$

Hence $y^{2}+(r-s)^{2} k^{2} \geqq 5^{-2}\left(y^{2}+(t-s)^{2} k^{2}\right) \geqq\left(4 \sqrt{2} C_{T}\right)^{2}$, which in turn implies, by the second inclusion of Lemma 2.7 , that $z(x, y)^{2}+y^{2}=x(r, s, y, k)^{2}+y^{2} \geqq$ $\left(2 C_{T}\right)^{2}$. We show next that the point of stationary phase is unique on the support of $A(x, z, y)$. Suppose that $z_{0}=x\left(r, s, y, k^{\prime}\right)$ is another point of stationary phase such that $z_{0}^{2}+y^{2} \geqq C_{T}^{2}$. Then, (2.26) and (2.27) imply that $x=x\left(t, s, y, k^{\prime}\right)$. But, such a $k$ is unique and we conclude that the stationary point is unique in $z^{2}+y^{2} \geqq C_{T}^{2}$. By definition of $S(t, s, x, y)$,

$$
S(t, r, x, x(r, s, y, k))+S(r, s, x(r, s, y, k), y)=S(t, s, x(t, s, y, k), y),
$$

which implies (2.43). The estimate (2.44) can be obtained as follows. We differentiate Eq. (2.42) repeatedly after replacing $z=z(x, y)$ and solve the resulting equation for $\partial_{x}^{\alpha} \partial_{y}^{\beta} z(x, y)$. Then, with the help of the induction argument, the non-singularity (2.35) of $\partial_{z}^{2} \phi(x, z, y)$ and the bounds (2.34) on the derivatives of $\partial_{z} \phi(x, z, y)$, we obtain the desired estimates. We omit the routine details.

We take $w \in C_{0}^{\infty}\left(\mathbf{R}^{m}\right)$ such that $w(z)=1$ for $|z| \leqq C_{T} / 4$ and $w(z)=0$ for $|z| \geqq C_{T} / 2$, and split the integral (2.40),

$$
\begin{aligned}
E_{2}(t, s, x, y)= & E_{2,1}(t, s, x, y)+E_{2,2}(t, s, x, y) \\
\equiv & \int e^{i \phi(x, z, y)} w(z-z(x, y)) A(x, z, y) d z \\
& \quad+\int e^{i \phi(x, z, y)}(1-w(z-z(x, y))) A(x, z, y) d z .
\end{aligned}
$$

For $x^{2}+y^{2} \geqq C_{7}^{2}$, Lemma 2.10 and definition of $w(z)$ implies that the support of $w(z-z(x, y))$ is contained in the $y^{2}+z^{2} \geqq\left(3 C_{T} / 2\right)^{2}$. Thus the integral $E_{2,1}(t, s, x, y)$ may be computed and estimated in entirely the same way as in the proof of Lemma 3.2 of [1], and one concludes easily that $e^{-i S} E_{2,1}$ satisfies the desired properties. We do not repeat the arguments here. 
To analyze $e^{-i S} E_{2,2}$ we write, using the notation $z_{0}=z(x, y)$,

$$
e^{-i S(t, s, x, y)} E_{2,2}(t, s, x, y)=\int e^{i\left(\phi(x, z, y)-\phi\left(x, z_{0}, y\right)\right)}\left(1-w\left(z-z_{0}\right)\right) A(x, z, y) d z .
$$

This will be treated below via integration by parts following basically the same line presented in [1]. Because the phase and the amplitude functions are defined and estimated only on the exterior of a compact set, however, we need two estimates, presented in the following lemma, which replace the key estimates, (3.13) and (3.47) of [1], used in the corresponding part of the argument.

Lemma 2.11. Let $(x, z, y)$ satisfy $x^{2}+y^{2} \geqq C_{7}^{2}$ and $y^{2}+z^{2} \geqq C_{T}^{2}$. Then

$$
\left|\left(\partial_{z} \phi\right)(x, z, y)\right| \geqq G|z-z(x, y)| / 2,
$$

where $G=(t-r)^{-1}+(r-s)^{-1}$, and

$\left|\partial_{x}^{\alpha} \partial_{y}^{\beta}(\phi(x, z, y)-\phi(x, z(x, y), y))\right| \leqq C_{\alpha \beta}(1+|z-z(x, y)|), \quad|\alpha+\beta| \geqq 1$.

Proof. To prove (2.48), we connect $z$ and $z(x, y)$ by a curve $\gamma(\tau), 0 \leqq \tau \leqq 1$, of length $\leqq \pi|z-z(x, y)|$ which satisfies $y^{2}+\gamma(\tau)^{2} \geqq C_{T}^{2}$ so that $\phi(x, \gamma(\tau), y)$ is well defined. Since $z(x, y)$ is a point of stationary phase, $\left(\partial_{z} \phi\right)(x, z(x, y), y)=0$, it follows that

$$
\begin{aligned}
& \left(\partial_{z} \phi\right)(x, z, y)=\left(\partial_{z} \phi\right)(x, z, y)-\left(\partial_{z} \phi\right)(x, z(x, y), y) \\
& \quad=\int_{0}^{1}\left(\left(\partial_{z}^{2} \phi\right)(x, \gamma(\tau), y)-G\right) \dot{\gamma}(\tau) d \tau+G(z-z(x, y)) .
\end{aligned}
$$

Relation (2.35) now readily implies (2.48), as well as the upper bound

$$
\left|\left(\partial_{z} \phi\right)(x, z, y)\right| \leqq 3 G|z-z(x, y)| / 2 .
$$

We prove (2.49). Let $|y| \geqq 2 C_{T}$. Then, for any $(x, z) \in \mathbf{R}^{2 m},(x, z, y)$ is in the domain of definition of $\phi(x, z, y)$, and

$\phi(x, z, y)-\phi(x, z(x, y), y)=\int_{0}^{1}\left(\partial_{z} \phi\right)(x, \tau z+(1-\gamma) z(x, y), y) d \tau \cdot(z-z(x, y))$.

Differentiate (2.52) and apply (2.34), (2.44) and (2.51). Estimate (2.49) follows. Next let $|y| \leqq 2 C_{T}$. We set $\Omega_{j}^{ \pm}=\left\{y=\left(y_{1}, \ldots, y_{m}\right): \pm y_{j} \geqq 0\right\}, j=1, \ldots, m$ and cover $\mathbf{R}^{m}$ by these sets. When $y \in \Omega_{j}^{ \pm}$, we telescope the difference $\phi(x, z, y)$ $\phi(x, z(x, y), y)$ as follows:

$$
\begin{aligned}
& \phi(x, z, y)-\phi(x, z(x, y), y)=\left(\phi(x, z, y)-\phi\left(x, z, y \pm 2 C_{T} e_{j}\right)\right) \\
& \quad+\left(\phi\left(x, z, y \pm 2 C_{T} e_{j}\right)-\phi\left(x, z\left(x, y \pm 2 C_{T} e_{j}\right), y \pm 2 C_{T} e_{j}\right)\right) \\
& \quad+\left(\phi\left(x, z\left(x, y \pm 2 C_{T} e_{j}\right), y \pm 2 C_{T} e_{j}\right)-\phi(x, z(x, y), y)\right)=I_{1}+I_{2}+I_{3}
\end{aligned}
$$

where $e_{j}$ is the $j^{\text {th }}$ unit vector. Note that for $y \in \Omega_{j}^{ \pm}$and $\pm \tau \geqq 0$, we clearly have $C_{T}^{2} \leqq z^{2}+y^{2} \leqq z^{2}+\left(y \pm \tau e_{j}\right)^{2}$ and $z^{2}+\left(y \pm 2 C_{T} e_{j}\right)^{2} \geqq\left(2 C_{T}\right)^{2}$. Hence, $I_{1}$ and 
$I_{3}$ are uniformly bounded with uniformly bounded derivatives with respect to $(x, y)$, as can be easily seen from the expressions

$$
I_{1}=-\int_{0}^{ \pm \sigma}\left(\partial_{y_{j}} \phi\right)\left(x, z, y+\tau e_{j}\right) d \tau,
$$

and

$$
\begin{aligned}
I_{3}= & \int_{0}^{ \pm \sigma}\left\{\left(\partial_{z} \phi\right)\left(x, z\left(x, y+\tau e_{j}\right), y+\tau e_{j}\right) \partial_{y_{j}} z\left(x, y+\tau e_{j}\right)\right. \\
& \left.+\left(\partial_{y_{j}} \phi\right)\left(x, z\left(x, y+\tau e_{j}\right), y+\tau e_{j}\right)\right\} d \tau .
\end{aligned}
$$

Applying to $I_{2}$ the estimate already proven for the case $|y| \geqq 2 C_{T}$, we obtain

$$
\left|\partial_{x}^{\alpha} \partial_{y}^{\beta} I_{2}\right| \leqq C_{\alpha \beta}\left(1+\left|z-z\left(x, y+2 C_{T} e_{j}\right)\right|\right) \text {. }
$$

But, as $z(x, y)$ has uniformly bounded derivatives, this immediately implies (2.49) for $I_{2}$. The proof follows by combining the above estimates.

Completion of the Proof of Theorem 1.1. We are now ready to show that the function $e^{-i S(t, s, x, y)} E_{2,2}(t, s, x, y)$ is smooth and bounded with respect to $(x, y)$ and that the derivatives are bounded and continuous with respect to $(t, s, x, y)$. Note, by virtue of (2.48) of the previous lemma,

$$
\left|\partial_{z} \phi(x, y, z)\right| \geqq C|z-z(x, y)| \geqq C C_{T} / 4,
$$

on the support of $1-w(z-z(x, y))$. This means that we can use the same operator $L$ defined by (2.39) to perform integration by parts in (2.47), which gives

$$
\begin{aligned}
e^{-i S(t, s, x, y)} E_{2,2}(t, s, x, y)= & \int e^{i(\phi(x, z, y)-\phi(x, z(x, y), y))}\left({ }^{t} L\right)^{\ell} \\
& \times((1-w(z-z(x, y))) A(x, z, y)) d z .
\end{aligned}
$$

Here, the lower bound (2.53) and the upper bound for the derivatives of $A(x, z, y)$, $z(x, y)$ and $\partial_{z} \phi(x, z, y)$ show that

$$
\left|\left(\partial_{x}^{\alpha} \partial_{y}^{\beta}\right)\left({ }^{t} L\right)^{\ell}(1-w(z-z(x, y))) A(x, z, y)\right| \leqq C_{\alpha \beta}(1+|z-z(x, y)|)^{-\ell} .
$$

Hence differentiating (2.54) with respect to $(x, y)$ under the integral sign and using the estimates (2.49), we see that $e^{-i S(t, s, x, y)} E_{2,2}(t, s, x, y)$ is bounded with bounded $(x, y)$ derivatives which are jointly continuous with respect to $(t, s, x, y)$.

To complete the proof, we show finally that the derivatives $E_{\alpha \beta}=\partial_{x}^{\alpha} \partial_{y}^{\beta} E(t, s, x, y)$ are $C^{\sigma+1}$ with respect to $(t, s, x, y)$, provided that, for all $\alpha, \partial_{x}^{\alpha} V(t, x)$ are $C^{\sigma}$ with respect to $(t, x)$. For this we show that $E(t, s, x, y)$ satisfies the following equations in the sense of distributions:

$$
\begin{gathered}
i \partial_{t} E(t, s, x, y)=-(1 / 2) \triangle_{x} E(t, s, x, y)+V(t, x) E(t, s, x, y), \\
i \partial_{s} E(t, s, x, y)=(1 / 2) \triangle_{y} E(t, s, x, y)-V(s, y) E(t, s, x, y) .
\end{gathered}
$$

Once these equations are established, the $C^{\sigma+1}$ property of $E_{\alpha \beta}$ follows immediately. Indeed, by differentiating (2.55) and (2.56) by $\partial_{x}^{\alpha} \partial_{y}^{\beta}$ and applying the previous result that $E_{\alpha \beta}$ is jointly continuous, we deduce easily that $\partial_{t} E_{\alpha \beta}$ and $\partial_{s} E_{\alpha \beta}$ are both jointly continuous with respect to $(t, s, x, y)$. Further differentiation of (2.55) and (2.56) 
by $(t, s, x, y)$, and a standard induction argument now lead to the conclusion that $\partial_{x}^{\alpha} \partial_{y}^{\beta} E(t, s, x, y)$ is $C^{\sigma+1}$. To prove $(2.55)$, it suffices to show that

$$
\int_{\mathbf{R}^{2 m+2}}\left(i \partial_{t}+(1 / 2) \triangle_{x}-V(t, x)\right) \phi(t, x) \psi(s, y) \cdot E(t, s, x, y) d y d x d t d s=0,
$$

for $\phi(t, x), \psi(s, y) \in C_{0}^{\infty}\left(\mathbf{R}^{m+1}\right)$. But this follows immediately because the integral is absolutely convergent and, for every fixed $s \in \mathbf{R}^{1}$,

$$
u(t, x ; s) \equiv \int_{\mathbf{R}^{m}} E(t, s, x, y) \psi(s, y) d y
$$

is a solution of (1.1) by the definition of $E(t, s, x, y)$. The second Eq. (2.56) follows from the first (2.55) and the identity $E(t, s, x, y)=E(s, t, y, x)^{*}$, which follows in turn from the identity $U(s, t)=U(t, s)^{*}$, where the former $*$ denotes the complex conjugate and the latter $*$ the conjugate operator. This completes the proof of Theorem 1.1.

\section{Super-Quadratic Potentials - Proof of Theorem 1.2}

In this section, we prove Theorem 1.2. We assume the spatial dimension $m=1$ and $V(x)$ satisfies the condition of Theorem 1.2. Under this condition it is well known that the operator $H=-(1 / 2) d^{2} / d x^{2}+V(x)$ is essentially selfadjoint on $C_{0}^{\infty}\left(\mathbf{R}^{1}\right)$ and its closure, which we denote by the same symbol, has purely discrete spectrum $\lambda_{1}<\lambda_{2}<\cdots \rightarrow \infty$. We let $u_{1}(x), u_{2}(x), \ldots$ be the associated real normalized eigenfunctions.

We begin with the study of the asymptotic behavior of solutions of the eigenfunction equation

$$
-(1 / 2) u^{\prime \prime}(x)+V(x) u(x)=\lambda u(x)
$$

in the interval $\Omega_{\lambda}=\{x: V(x) \leqq \lambda / 2\}$, as the parameter $\lambda \rightarrow \infty$. We set

$$
S(x)=\int_{0}^{x} \sqrt{2(\lambda-V(s))} d s, \quad a(x)=(2(\lambda-V(x)))^{-1 / 4},
$$

and make the well known change of variables (cf. Titchmarsh [17], p. 119): $x \rightarrow$ $y=S(x)$ and $u(x)=a(x) w(y)$. This transforms the eigen-Eq. (3.1) into

$$
\frac{d^{2} w}{d y^{2}}+w+\left\{\frac{V^{\prime \prime}(x)}{8(\lambda-V(x))^{2}}+\frac{5 V^{\prime}(x)^{2}}{32(\lambda-V(x))^{3}}\right\} w=0, \quad y=S(x) .
$$

It follows that $w$ satisfies the integral equation:

$$
w(y)=w(0) \cos y+w^{\prime}(0) \sin y-\int_{0}^{y} \sin (y-z) V(z, \lambda) w(z) d z
$$

where, of course, $V(y, \lambda)$ stands for the function inside $\{\cdots\}$,

$$
V(y, \lambda)=\frac{V^{\prime \prime}(x)}{8(\lambda-V(x))^{2}}+\frac{5 V^{\prime}(x)^{2}}{32(\lambda-V(x))^{3}}, \quad y=S(x) .
$$

We write $C_{\lambda}=\left(w(0)-i w^{\prime}(0)\right)$ so that $w(0) \cos y+w^{\prime}(0) \sin y=\operatorname{Re}\left(C_{\lambda} e^{i y}\right)$. 
Lemma 3.1. There exists a constant $C>0$ independent of $\lambda \geqq \lambda_{0}$ and $x \in \Omega_{\lambda}$ such that

$$
\left|a(x)^{-1} u(x)-\operatorname{Re}\left\{C_{\lambda} e^{i S(x)}\right\}\right| \leqq C\left|C_{\lambda}\right| \lambda^{-1 / 2} .
$$

Proof. Let $\left(x_{0}, x_{1}\right) \subset \Omega_{\lambda} \backslash K$. We assume without loss of generality that $0<x_{0}<$ $x_{1}$ and $V^{\prime}(x) \geqq 0$ on the interval. Since $V^{\prime} \leqq C V(x) \leqq C(\lambda-V(x))$, we have, uniformly with respect to $x_{0}$ and $x_{1}$, that

$$
\int_{S\left(x_{0}\right)}^{S\left(x_{1}\right)} V(y, \lambda) d y \leqq \frac{C}{\lambda^{3 / 2}} \int_{x_{0}}^{x_{1}} V^{\prime \prime}(x) d x+\int_{x_{0}}^{x_{1}} \frac{C V^{\prime}(x)}{(\lambda-V(x))^{3 / 2}} d x \leqq \frac{C}{\lambda^{1 / 2}} .
$$

It follows by applying Gronwall's inequality to (3.2) that $|w| \leqq C\left|C_{\lambda}\right|$, and inserting this inequality into (3.2) and using (3.4) again, we obtain (3.3).

We have $S(x)=\sqrt{2 \lambda} x+O\left(\lambda^{-1 / 2}\right)$ and $a(x)=(2 \lambda)^{-1 / 4}\left(1+O\left(\lambda^{-1}\right)\right)$ uniformly on any compact set $I$. Hence, as $\lambda \rightarrow \infty$, we have

$$
u(x)=(2 \lambda)^{-1 / 4}\left(\operatorname{Re}\left\{C_{\lambda} e^{i \sqrt{2 \lambda} x}\right\}+O\left(C_{\lambda} \lambda^{-1 / 2}\right)\right), \quad x \in I .
$$

We now assume that the solution $u$ belongs to $L^{2}\left(\mathbf{R}^{1}\right)$ and find a lower bound of $\left|C_{\lambda}\right|$ for large $\lambda$. In what follows $(\cdot, \cdot)$ denotes the inner product on $L^{2}\left(\mathbf{R}^{1}\right)$.

Lemma 3.2. Let a real function $u \in L^{2}\left(\mathbf{R}^{1}\right)$ satisfy (3.1) and suppose $\|u\|=1$. Then, there exists a constant $C>0$ such that $\left|C_{\lambda}\right| \geqq C \lambda^{(c-1) / 4 c}$ for $\lambda \geqq \lambda_{0}$.

Proof. We denote $M=\inf _{x \in K}\left(V(x)+(1 / 2) x V^{\prime}(x)\right)$. Let $A=(1 / 2)(x \cdot D+D \cdot x)$ be the generator of the spatial dilation $e^{i t A} u(x)=e^{t / 2} u\left(e^{t} x\right)$. By a simple computation we have

$$
i[H, A]=2 H-\left(2 V+x V^{\prime}\right),
$$

where $[H, A]=H A-A H$ is the commutator. Since $u(x)$ is $C^{5}$ and exponentially decreasing as $x \rightarrow \pm \infty$ with its derivatives, integration by parts shows that

$$
([H, A] u, u)=(A u, H u)-(H u, A u)=\lambda\{(A u, u)-(u, A u)\}=0
$$

and we obtain the virial identity,

$$
\lambda=(H u, u)=\left(\left(V+(1 / 2) x \cdot V^{\prime}\right) u, u\right) .
$$

For large $\lambda_{0}$ we have $K \subset \Omega_{\lambda}$ for $\lambda \geqq \lambda_{0}$. Hence, by the assumption $2 c V(x) \leqq$ $x V^{\prime}(x)$ on $K^{c}$,

$$
\begin{aligned}
\lambda & \geqq M+\int_{K^{c}}\left(V(x)+(1 / 2) x \cdot V^{\prime}(x)\right)|u(x)|^{2} d x \\
& \geqq M+\int_{\Omega_{\lambda}^{c}}(1+c) V(x)|u(x)|^{2} d x \geqq M+\frac{\lambda(1+c)}{2} \int_{\Omega_{\lambda}^{c}}|u(x)|^{2} d x .
\end{aligned}
$$

It follows that for large $\lambda \geqq \lambda_{0}$,

$$
\int_{\Omega_{\lambda}}|u(x)|^{2} d x=1-\int_{\Omega_{\lambda}^{c}}|u(x)|^{2} d x \geqq \frac{(c-1) \lambda+2 M}{(c+1) \lambda} \geqq \frac{1}{2} \frac{(c-1)}{(c+1)} .
$$


On the other hand (3.3) implies $u(x)=a(x)\left(\operatorname{Re}\left\{C_{\lambda} e^{i S(x)}\right\}+O\left(\left|C_{\lambda}\right| \lambda^{-1 / 2}\right)\right)$ on $\Omega_{\lambda}$ and

$$
\int_{\Omega_{\lambda}}|u(x)|^{2} d x=\int_{\Omega_{\lambda}} \frac{\left|\operatorname{Re}\left\{C_{\lambda} e^{i S(x)}\right\}+O\left(\left|C_{\lambda}\right| \lambda^{-1 / 2}\right)\right|^{2}}{\sqrt{2(\lambda-V(x))}} d x \leqq \frac{\left|C_{\lambda}\right|^{2}}{\sqrt{\lambda}}\left|\Omega_{\lambda}\right|\left(1+C \lambda^{-1 / 2}\right),
$$

where $\left|\Omega_{\lambda}\right|$ is the measure of $\Omega_{\lambda}$. On the other hand, the assumptions imply $V(x) \geqq$ $C|x|^{2 c}$ outside a compact set and we have $\left|\Omega_{\lambda}\right| \leqq C \lambda^{1 / 2 c}$. It follows by combining (3.6) with (3.7) that $\left|C_{\lambda}\right| \geqq C\left|\Omega_{\lambda}\right|^{-1 / 2} \lambda^{1 / 4} \geqq C^{\prime} \lambda^{(c-1) / 4 c}$ as desired.

We now show that the spacing of the neighboring eigenvalues $\lambda_{n+1}-\lambda_{n}$ is algebraically increasing with $\lambda_{n}$ as $n \rightarrow \infty$. From the assumption on $V$ we deduce that, outside a compact interval $K, V(x)$ is increasing with respect to $|x|$ and $C|x|^{2 c} \leqq V(x) \leqq C^{\prime}|x|^{2 d}$ for some $d \geqq c$ outside a compact set. It follows for large $\lambda_{n}$, that the equation $V(x)=\lambda_{n}$ has two roots $X_{n}^{\prime}<0<X_{n}$. It is well known (cf. Titchmarsh [17], p. 151) that

$$
\frac{1}{\pi_{X_{n}^{\prime}}} \int_{n}^{X_{n}}\left\{2\left(\lambda_{n}-V(x)\right)\right\}^{1 / 2} d x=n+\frac{1}{2}+O\left(\frac{1}{n}\right) .
$$

Lemma 3.3. There exists a constant $C>0$ such that for large $n$, we have

$$
\left|\lambda_{n \pm 1}-\lambda_{n}\right| \geqq C \lambda_{n}^{1 / 2-1 / 2 c}
$$

Proof. Without loss of generality, we may assume $\min V(x)=0$. For large $n$, we have $X_{n}<X_{n+1}, X_{n}^{\prime}>X_{n+1}^{\prime}$ and $C^{\prime} \lambda_{n}^{1 / 2 d} \leqq X_{n}-X_{n}^{\prime} \leqq C \lambda_{n}^{1 / 2 c}$. Subtracting (3.8) from both sides of the corresponding equation for $n+1$, we obtain

$$
\begin{aligned}
\pi+ & O\left(\frac{1}{n}\right)=\int_{X_{n}^{\prime}}^{X_{n}}\left(\left\{2\left(\lambda_{n+1}-V(x)\right)\right\}^{1 / 2}-\left\{2\left(\lambda_{n}-V(x)\right)\right\}^{1 / 2}\right) d x \\
+ & \left(\int_{X_{n+1}^{\prime}}^{X_{n}^{\prime}}+\int_{X_{n}}^{X_{n+1}}\right)\left\{2\left(\lambda_{n+1}-V(x)\right)\right\}^{1 / 2} d x \equiv I_{1}+I_{2}+I_{3} .
\end{aligned}
$$

Obviously, $0 \leqq I_{j} \leqq 2 \pi$ for large $n$. Estimating $I_{1}$ from below, we have

$$
I_{1}=\int_{X_{n}^{\prime}}^{X_{n}} \frac{2\left(\lambda_{n+1}-\lambda_{n}\right)}{\left\{2\left(\lambda_{n+1}-V(x)\right)\right\}^{1 / 2}+\left\{2\left(\lambda_{n}-V(x)\right)\right\}^{1 / 2}} d x \geqq \frac{\left(\lambda_{n+1}-\lambda_{n}\right)\left|X_{n}-X_{n}^{\prime}\right|}{\sqrt{2 \lambda_{n+1}}} .
$$

It follows that $\lambda_{n+1}-\lambda_{n} \leqq 2 \pi \sqrt{2 \lambda_{n+1}}\left|X_{n}-X_{n}^{\prime}\right|^{-1} \leqq C \lambda_{n+1}^{1 / 2} \lambda_{n}^{-1 / 2 d}$ and

$$
0 \leqq 1-\lambda_{n} / \lambda_{n+1} \leqq C \lambda_{n+1}^{-1 / 2} \lambda_{n}^{-1 / 2 d} \rightarrow 0, \quad n \rightarrow \infty \text {. }
$$

(Incidentally this estimate yields the upper bound $\lambda_{n+1}-\lambda_{n} \leqq C \lambda_{n}^{1 / 2-1 / 2 d}$.) On the other hand, if $Y_{n}^{\prime}<0<Y_{n}$ are the two roots of $V(x)=\lambda_{n} / 2$, we have

$$
\frac{I_{1}}{\lambda_{n+1}-\lambda_{n}} \leqq \int_{Y_{n}^{\prime}}^{Y_{n}} \frac{1}{\lambda_{n}^{1 / 2}} d x+\left(\int_{X_{n}^{\prime}}^{Y_{n}^{\prime}}+\int_{Y_{n}}^{X_{n}}\right) \frac{V^{\prime}(x)}{\left\{2\left(\lambda_{n}-V(x)\right)\right\}^{1 / 2}} \frac{x d x}{x V^{\prime}(x)} .
$$


Estimating the second integral by using $x V^{\prime}(x) \geqq 2 c V(x) \geqq c \lambda_{n}$ and $|x| \leqq C \lambda_{n}^{1 / 2 c}$, we bound the right hand side by

$$
\frac{Y_{n}-Y_{n}^{\prime}}{\lambda_{n}^{1 / 2}}+\frac{C \lambda_{n}^{1 / 2 c}}{c \lambda_{n}}\left(-\int_{X_{n}^{\prime}}^{Y_{n}^{\prime}}+\int_{Y_{n}}^{X_{n}}\right) \frac{V^{\prime}(x) d x}{\left\{2\left(\lambda_{n}-V(x)\right)\right\}^{1 / 2}}=\frac{Y_{n}-Y_{n}^{\prime}}{\lambda_{n}^{1 / 2}}+\frac{C \lambda_{n}^{1 / 2 c}}{c \lambda_{n}} \lambda_{n}^{1 / 2} .
$$

This yields the estimate $I_{1} \leqq C\left(\lambda_{n+1}-\lambda_{n}\right) \lambda_{n}^{1 / 2 c-1 / 2}$. We estimate $I_{2}$ and $I_{3}$ in a similar fashion. By using $x V^{\prime}(x) \geqq 2 c V(x) \geqq 2 c \lambda_{n}$ and $|x| \leqq C \lambda_{n+1}^{1 / 2 c} \leqq C^{\prime} \lambda_{n}^{1 / 2 c}$ which are valid on the region of integration, we see that

$$
\begin{aligned}
I_{3} & =\int_{X_{n}}^{X_{n+1}} \frac{\left\{2\left(\lambda_{n+1}-V(x)\right)\right\}^{1 / 2} x V^{\prime}(x)}{x V^{\prime}(x)} d x \leqq C \frac{\lambda_{n}^{1 / 2 c}}{\lambda_{n}} \int_{X_{n}}^{X_{n+1}}\left\{2\left(\lambda_{n+1}-V(x)\right)\right\}^{1 / 2} V^{\prime}(x) d x \\
& =\frac{2 \sqrt{2} C}{3}\left(\lambda_{n+1}-\lambda_{n}\right) \lambda_{n}^{1 / 2 c-1 / 2} \cdot\left(\frac{\lambda_{n+1}}{\lambda_{n}}-1\right)^{1 / 2} \leqq C_{n}\left(\lambda_{n+1}-\lambda_{n}\right) \lambda_{n}^{1 / 2 c-1 / 2}
\end{aligned}
$$

with the constant $C_{n} \rightarrow 0$ as $n \rightarrow \infty$. We may estimate $I_{2}$ in entirely the same way and arrive at

$$
\pi+O\left(\frac{1}{n}\right)=I_{1}+I_{2}+I_{3} \leqq C\left(\lambda_{n+1}-\lambda_{n}\right) \lambda_{n}^{1 / 2 c-1 / 2},
$$

which clearly implies $\lambda_{n+1}-\lambda_{n} \geqq C \lambda_{n}^{1 / 2-1 / 2 c}$. This completes the proof of the lemma since $\lambda_{n} / \lambda_{n-1} \rightarrow 1$ as $n \rightarrow \infty$.

Proof of Theorem 1.2. It suffices to show that for any non-negative and not identically vanishing $C_{0}^{\infty}$ functions $\phi, \Phi$ and $\Psi$,

$$
\int_{\mathbf{R}^{3}} E(t, x, y) \phi(t) \Phi(x) \Psi(y) e^{i(t \lambda+x \cdot \xi-y \cdot \eta)} d t d x d y
$$

is bounded from below in modulus by a constant times $(\lambda+|\xi|+|\eta|)^{-1 / 2 c}$, along a sequence $\left(\lambda_{n}, \xi_{n}, \eta_{n}\right)$ such that $\lambda_{n}+\left|\xi_{n}\right|+\left|\eta_{n}\right| \rightarrow \infty, n \rightarrow \infty$. We write $\Phi_{\xi}(x)=$ $\Phi(x) e^{i x \cdot \xi}$ and $\Psi_{\eta}(x)=\Psi(x) e^{i x \cdot \eta}$. By the definition of $E(t, x, y)$, (3.11) may be written as

$$
\int_{-\infty}^{\infty}\left(e^{-i t(H-\lambda)} \Phi_{\xi}, \Psi_{\eta}\right) d t=\left(\widehat{\phi}(\lambda-H) \Phi_{\xi}, \Psi_{\eta}\right)
$$

where $\widehat{\phi}$ is the Fourier transform of $\phi$ :

$$
\widehat{\phi}(\lambda)=\int_{-\infty}^{\infty} e^{-i t \lambda} \phi(t) d t .
$$

Let $\lambda_{n}$ be the $n^{\text {th }}$ eigenvalue of $H$ and $P_{n}$ denote the orthogonal projection onto the eigenspace spanned by $u_{n}$ and let $Q_{n}=1-P_{n}$. By virtue of Lemma 3.3 and the fact that $\widehat{\phi}$ is rapidly decreasing, we have for any $N=1,2, \ldots$,

$$
\left\|\widehat{\phi}\left(\lambda_{n}-H\right)-\widehat{\phi}(0) P_{n}\right\|=\left\|\widehat{\phi}\left(\lambda_{n}-H\right) Q_{n}\right\| \leqq \sup _{k \neq n}\left|\widehat{\phi}\left(\lambda_{n}-\lambda_{k}\right)\right| \leqq C_{N} \lambda_{n}^{-N(c-1) / 2 c},
$$

with a constant $C_{N}$ independent of $n=1,2, \ldots$ It follows that for any large $N$ we have, uniformly with respect to $\xi$ and $\eta$,

$$
\left(\widehat{\phi}\left(H-\lambda_{n}\right) \Phi_{\xi}, \Psi_{\eta}\right)=\widehat{\phi}(0)\left(\Phi_{\xi}, u_{n}\right)\left(u_{n}, \Psi_{\eta}\right)+O\left(\lambda_{n}^{-N}\right)
$$


as $n \rightarrow \infty$. On the other hand (3.5) and Lemma 3.2 show that

$$
\left(\Phi_{\sqrt{2 \lambda_{n}}}, u_{n}\right)=\frac{1}{2\left(2 \lambda_{n}\right)^{1 / 4}}\left\{\int \Phi(x)\left(C_{\lambda_{n}} e^{2 i \sqrt{2 \lambda_{n}} x}+\overline{C_{\lambda_{n}}}\right) d x+O\left(C_{\lambda_{n}} \lambda_{n}^{-1 / 2}\right)\right\},
$$

and integration by parts yields

$$
\left(\Phi_{\sqrt{2 \lambda_{n}}}, u_{n}\right)=\frac{\overline{C_{\lambda_{n}}}}{2\left(2 \lambda_{n}\right)^{1 / 4}}\left(\int \Phi(x) d x+O\left(\lambda_{n}^{-1 / 2}\right)\right) .
$$

We may compute $\left(u_{n}, \Psi_{\sqrt{2 \lambda_{n}}}\right)$ in a similar fashion and conclude that

$$
\left(\widehat{\phi}\left(H-\lambda_{n}\right) \Phi_{\sqrt{2 \lambda_{n}}}, \Psi_{\sqrt{2 \lambda_{n}}}\right)=\frac{\left|C_{\lambda_{n}}\right|^{2}}{4\left(2 \lambda_{n}\right)^{1 / 2}}\left(\widehat{\phi}(0) \widehat{\Phi}(0) \widehat{\Psi}(0)+O\left(\lambda_{n}^{-1 / 2}\right)\right)+O\left(\lambda_{n}^{-N}\right) .
$$

Thus, setting $\lambda=\lambda_{n}$ and $\xi=\eta=\sqrt{2 \lambda_{n}}$ and using Lemma 3.2, we have obtained the desired lower bound along this sequence

$$
\left|\left(\widehat{\phi}(H-\lambda) \Phi_{\xi}, \Psi_{\eta}\right)\right| \geqq C(\lambda+|\xi|+|\eta|)^{-1 / 2 c} .
$$

This completes the proof of Theorem 1.2.

Proof of Remark 1. (1) We show that $E(t, x, y)$ is nowhere in $\mathscr{L}_{1, \text { loc }}^{1 / 2 c}\left(\mathbf{R}^{2}\right)$ with respect to $(t, y)$ for almost all $x \in \mathbf{R}^{1}$. The second statement follows from $E(t, x, y)=$ $E(-t, y, x)^{*}$. We use the notation of the proof of Theorem 1.2. It suffices to show that, for almost all $x \in \mathbf{R}^{1}$, $\lim \sup _{n \rightarrow \infty}$ of

$$
\lambda_{n}^{-1 / 2 c}\left|\int_{\mathbf{R}^{2}} E(t, x, y) \phi(t) \Phi(y) e^{i\left(t \lambda_{n}+y \sqrt{2 \lambda_{n}}\right)} d y d t\right|=\lambda_{n}^{-1 / 2 c}\left|\hat{\phi}\left(H-\lambda_{n}\right) \Phi_{\sqrt{2 \lambda_{n}}}(x)\right|
$$

is positive. Decompose, as before,

$$
\widehat{\phi}\left(H-\lambda_{n}\right) \Phi_{\sqrt{2 \lambda_{n}}}(x)=\widehat{\phi}(0)\left(P_{n} \Phi_{\sqrt{2 \lambda_{n}}}\right)(x)+\widehat{\phi}\left(H-\lambda_{n}\right)\left(Q_{n} \Phi_{\sqrt{2 \lambda_{n}}}\right)(x) .
$$

The second term on the right may be estimated as follows by using the Sobolev inequality,

$$
\sup _{x \in \mathbf{R}}\left|\widehat{\phi}\left(H-\lambda_{n}\right)\left(Q_{n} \Phi_{\sqrt{2 \lambda_{n}}}\right)(x)\right|^{2} \leqq C\left\|\widehat{\phi}\left(H-\lambda_{n}\right) Q_{n} \Phi_{\sqrt{2 \lambda_{n}}}\right\|_{H^{1}}^{2} .
$$

Choose a constant $M>0$ such that $V(x)+M \geqq 1 / 2$ and estimate the right handside by

$$
2 C\left((H+M) \widehat{\phi}\left(H-\lambda_{n}\right) Q_{n} \Phi_{\sqrt{2 \lambda_{n}}}, \widehat{\phi}\left(H-\lambda_{n}\right) Q_{n} \Phi_{\sqrt{2 \lambda_{n}}}\right),
$$

which is further estimated as in the proof of Theorem 1.2, to give the bound

$$
\begin{aligned}
\left(\left\|\left(H-\lambda_{n}\right) \widehat{\phi}\left(H-\lambda_{n}\right) Q_{n}\right\|+\right. & \left.\left\|\left(\lambda_{n}+M\right) \widehat{\phi}\left(H-\lambda_{n}\right) Q_{n}\right\|\right) \cdot\left\|\widehat{\phi}\left(H-\lambda_{n}\right) Q_{n}\right\|\|\Phi\|^{2} \\
& \leqq C_{N} \lambda_{n}^{-N},
\end{aligned}
$$

where $N$ is an arbitrarily large number. If we write $\operatorname{Re} C_{n} e^{i \sqrt{2 \lambda_{n}} x}=\left|C_{n}\right| \cos \left(\sqrt{2 \lambda_{n}} x+\right.$ $\left.\tau_{n}\right)$, the asymptotic formulae (3.5) and (3.13) imply for the first term of (3.14)

$$
\begin{aligned}
\widehat{\phi}(0)\left(P_{n} \Phi_{\sqrt{2 \lambda_{n}}}\right)(x)= & \frac{\widehat{\phi}(0)}{2 \sqrt{2 \lambda_{n}}}\left|C_{\lambda_{n}}\right|^{2} e^{-i \tau_{n}}\left\{\cos \left(\sqrt{2 \lambda_{n}} x+\tau_{n}\right)\right. \\
& \left.+O\left(\lambda_{n}^{-1 / 2}\right)\right\}\left(\hat{\Phi}(0)+O\left(\lambda_{n}^{-1 / 2}\right)\right) .
\end{aligned}
$$


Thus, if we set $G_{L, n, \ell}=\left\{|x| \leqq L:\left|\cos \left(\sqrt{2 \lambda_{n}} x+\tau_{n}\right)\right|>2^{-\ell}\right\}$ and $G_{L}=$ $\bigcup_{\ell=1}^{\infty} \bigcap_{k=1}^{\infty} \bigcup_{n=k}^{\infty} G_{L, n, \ell}$, we have

$$
\lim \sup _{n \rightarrow \infty} \lambda_{n}^{1 / 2 c}\left|\widehat{\phi}\left(H-\lambda_{n}\right) \Phi_{\sqrt{2 \lambda_{n}}}(x)\right|>0, \quad \text { for all } x \in G_{L} .
$$

But the complement $G_{L, n, \ell}^{c}$ of $G_{L, n, \ell}$ in the interval $[-L, L]$ has a measure $\leqq C L 2^{-\ell}$ for large $\ell$ and $n$, and the measure of the set $G_{L}^{c}=\bigcap_{\ell=1}^{\infty} \bigcup_{k=1}^{\infty} \bigcap_{n=k}^{\infty} G_{L, n, \ell}^{c}$ vanishes. This proves statement (1).

Proof of Remark 1. (2) The function $E(t, x, y)$ satisfies the Schrödinger equations

$$
\left(i \partial_{t}+(1 / 2) \triangle_{x}-V(t, x)\right) E(t, x, y)=\left(i \partial_{t}+(1 / 2) \triangle_{y}-V(t, y)\right) E(t, x, y)=0,
$$

as can be easily checked as in the proof (2.55) and (2.56). Then, the theory of Lascar [12] and Sakurai [15] on the propagation of the quasi-homogeneous wave front sets implies that

$$
W F^{a} E \subset\left\{(t, x, y, \lambda, \eta, \xi): 2 \lambda=\xi^{2}=\eta^{2}\right\},
$$

viz, for any $\left(t_{0}, x_{0}, y_{0}\right)$ there exist $\rho(t), \psi(x), \phi(y)$ such that $\Phi(t, x, y)=\rho(t) \psi(x) \phi(y)$ does not vanish at this point and, for any small $\varepsilon>0$, $\widehat{\Phi E}$ decays rapidly outside the parabolic set

$$
\left\{(\lambda, \eta, \xi) \in \mathbf{R}^{3}:|1-| \xi|/| \eta||+\left|1-2 \lambda / \xi^{2}\right|+\left|1-2 \lambda / \eta^{2}\right| \leqq \varepsilon\right\},
$$

as follows: for any large $N,|\widehat{\Phi E}(\lambda, \eta, \xi)| \leqq C_{N}\left(1+|\lambda|+|\eta|^{2}+|\xi|^{2}\right)^{-N}$. It follows that we have only to estimate

$$
\begin{aligned}
\widehat{\Phi E}(\lambda, \eta,-\xi) & =\left(\hat{\rho}(H-\lambda) \phi_{\xi}, \psi_{\eta}\right) \\
& =\left(\hat{\rho}(H-\lambda) P_{n} \phi_{\xi}, \psi_{\eta}\right)+\left(\hat{\rho}(H-\lambda) Q_{n} \phi_{\xi}, \psi_{\eta}\right)
\end{aligned}
$$

in the set (3.15). If $\lambda_{n}$ is the eigenvalue of $H$ nearest to $\lambda$, we have $\lambda \in$ $\left(\lambda_{n-1}, \lambda_{n+1}\right)$ and the distance from $\lambda$ to the end points of this interval is greater than $(1 / 2) \min \left\{\lambda_{n+1}-\lambda_{n}, \lambda_{n}-\lambda_{n-1}\right\}$. Thus, by using Lemma 3.3 as in the proof of Theorem 1.2, we deduce that the second term in the right of (3.16) is bounded by

$$
\left|\left(\hat{\rho}(H-\lambda) Q_{n} \phi_{\xi}, \psi_{\eta}\right)\right| \leqq C_{N} \lambda^{-N} \leqq C_{N}\left(1+|\lambda|+|\eta|^{2}+|\xi|^{2}\right)^{-N} .
$$

On the other hand, when $\lambda$ is large enough, the asymptotic formula (3.5) implies

$$
\begin{aligned}
\left|\left(\hat{\rho}(0) P_{n} \phi_{\xi}, \psi_{\eta}\right)\right| & \leqq\|\rho\|_{L^{1}}\left|\left(\phi_{\xi}, u_{n}\right)\right|\left|\left(u_{n}, \psi_{\eta}\right)\right| \\
& \leqq C\left(2 \lambda_{n}\right)^{-1 / 2}\left|C_{\lambda_{n}}\right|^{2}\left|\Omega_{\lambda_{n}}\right|\|\rho\|_{L^{1}}\|\psi\|_{L^{1}}\|\phi\|_{L^{1}} .
\end{aligned}
$$

But, we may estimate the left hand side of (3.7) from below as

$$
1 \geqq \int_{\Omega_{\lambda}}|u(x)|^{2} d x=\int_{\Omega_{\lambda}} \frac{\left|\operatorname{Re}\left\{C_{\lambda} e^{i S(x)}\right\}+O\left(\left|C_{\lambda}\right| \lambda^{-1 / 2}\right)\right|^{2}}{\sqrt{2(\lambda-V(x))}} d x \geqq \frac{\left|C_{\lambda}\right|^{2}}{2 \sqrt{2 \lambda}}\left|\Omega_{\lambda}\right|\left(1-C \lambda^{-1 / 2}\right),
$$

and, because $\Omega_{\lambda} \geqq C \lambda^{1 / 2 c}$ by assumption, we obtain $\left|C_{\lambda_{n}}\right| \leqq C \lambda_{n}^{1 / 4-1 / 4 c}$. This implies

$$
|\widehat{\Phi E}(\lambda, \eta,-\xi)| \leqq C\left(1+|\lambda|+|\eta|^{2}+|\xi|^{2}\right)^{-1 / 2 c}
$$

as desired. 
Acknowledgement. The author would like to express sincere thanks to Professor Akira Kaneko who kindly explained to him the works of Lascar [12] and Sakurai [15].

\section{References}

1. Asada, K., Fujiwara, D.: On some oscillatory integral transformation in $L^{2}\left(\mathbf{R}^{n}\right)$. Jap. J. Math. 4, 299-361 (1978)

2. Cartan, E.: Leçons sur les invariants intégraux. Paris, A Herman, 1922

3. Coddington, E., Levinson, N.: The theory of ordinary differential equations. New York: McGraw-Hill, 1955

4. Craig, W., Kappeler, T., Strauss, W.: Microlocal dispersive smoothing for the Schrödinger equation. Comm. Pure and Appl. Math. 45, 769-860 (1996)

5. Fujiwara, D.: A construction of the fundamental solution for the Schrödinger equation. J. d'Analyse Math. 35, 41-96 (1979)

6. Fujiwara, D.: Remarks on the convergence of the Feynman path integrals. Duke Math. J. 47, 41-96 (1980)

7. Hörmander, L.: Linear partial differential operators. Vol. IV, Tokyo: Springer Verlag, 1984

8. Kapitanski, L., Rodnianski, I.: Regulated smoothing for Schrödinger evolution. Internat. Math. Research Notices 2, 45-54 (1996)

9. Kapitanski, L., Rodnianski, I., Yajima, K.: In preparation.

10. Kapitanski, L., Safarov, Y.: Dispersive smoothing for Schrödinger equations. Research letters 3, 77-91 (1996)

11. Kitada, H., Kumano-go, H.: A family of Fourier integral operators and the fundamental solution for a Schrödinger equation. Osaka J. Math. 18, 291-360 (1981)

12. Lascar, R.: Propagation des singularités des solutions d'équations pseudo-differentielles quasihomogènes. Ann. Inst. Fourier 27, 79-123 (1977)

13. Mehler, F.G.: Ueber die Entwicklung einer Function von beliebig vielen Variablen nach Laplaceschen Functionen höherer Ordnung. J. reine und angew. Math. 66, 161-176 (1866)

14. Ozawa, T.: Smoothing effects and dispersion of singularities for Schrödinger evolution group. Arch. Rat. Mech. Anal. 110, 165-186 (1990)

15. Sakurai, T.: Quasi-homogeneous wave front set and fundamental solution for Schrödinger operators. Scientific Papers of Coll. General Edu. 32, 1-13 (1982)

16. Taylor, M.E.: Pseudodifferential Operators. Princeton, New Jersey: Princeton Univ. Press, 1981

17. Titchmarsh, E.C.: Eigenfunction Expansions associated with second order differential operators, Part I. Second edition. Oxford: Oxford Univ. Press, 1969

18. Yajima, K.: Schrödinger evolution equations with magnetic fields. J. d'Analyse Math. 56, 29-76 (1991)

19. Yajima, K.: Existence of solutions for Schrödinger evolution equations. Commun. Math. Phys. 110, 415-426 (1987)

20. Yamazaki, M.: On the microlocal smoothing effect of dispersive partial differential equations I: Second order linear equation. Algebraic Analysis 11, 911-926 (1988)

21. Zelditch, S.: Reconstruction of singularities for solutions of Schrödinger equation. Commun. Math. Phys. 90, 1-26 (1983)

22. Zygmund, A.: On the Fourier coefficients and transforms of functions of two variables. Studia Math. 50, 189-201 (1974) 
